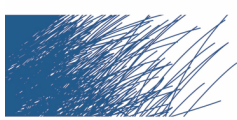

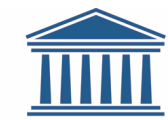 \\ UNIVERSITEIT \\ GENT
}

biblio.ugent.be

The UGent Institutional Repository is the electronic archiving and dissemination platform for all UGent research publications. Ghent University has implemented a mandate stipulating that all academic publications of UGent researchers should be deposited and archived in this repository. Except for items where current copyright restrictions apply, these papers are available in Open Access.

This item is the archived peer-reviewed author-version of:

Title: On the order of summability of the Fourier inversion formula

Authors: Jasson Vindas and Ricardo Estrada

In: Analysis in Theory and Applications 26 (1), 13-42, 2010

Optional: doi: 10.1007/s10496-010-0013-3

To refer to or to cite this work, please use the citation to the published version:

J. Vindas, R. Estrada (2010). On the order of summability of the Fourier inversion formula. Anal. Theory Appl. 26 (1) 13-42. doi: 10.1007/s10494-010-0013-3 


\title{
ON THE ORDER OF SUMMABILITY OF THE FOURIER INVERSION FORMULA
}

\author{
JASSON VINDAS AND RICARDO ESTRADA
}

\begin{abstract}
In this article we show that the order of the point value, in the sense of Łojasiewicz, of a tempered distribution and the order of summability of the pointwise Fourier inversion formula are closely related. Assuming that the order of the point values and certain order of growth at infinity are given for a tempered distribution, we estimate the order of summability of the Fourier inversion formula. For Fourier series, and in other cases, is shown that if the distribution has a distributional point value of order $k$, then its Fourier series is e.v. Cesàro summable to the distributional point value of order $k+1$. Conversely, we also show that if the pointwise Fourier inversion formula is e.v. Cesàro summable of order $k$, then the distribution is the $(k+1)$-th derivative of a locally integrable function, and the distribution has a distributional point value of order $k+2$. We also establish connections between orders of summability and local behavior for other Fourier inversion problems.
\end{abstract}

\section{INTRODUCTION}

The present article is concerned with the study of the summability of the pointwise Fourier inversion formula for tempered distributions reported in $[35,36]$.

The study of the relation between the value of a function at a point and the convergence and summability of Fourier series and Fourier integrals has a long history. Since the convergence fails in most interesting cases, this study is carry out by means of summability methods.

In the case of Fourier series, it was said by A. Zygmund [46] that the problem of summability of Fourier Series of classical functions at individual points could be considered as a closed chapter in Mathematics. However, since the introduction of the so called Generalized Functions, new problems were opened.

Using the concept of the value of a distribution at a point in the sense of Łojasiewicz [21], one can extend many results from the classical theory

2000 Mathematics Subject Classification. Primary 42A24, 42A38, 46F10. Secondary 40G05, 42A50, 46F12.

Key words and phrases. Fourier inversion formula, tempered distributions, distributional point values, Cesàro summability of Fourier series and integrals, summability of distributional evaluations, jump.

First author current address: Department of Pure Mathematics and Computer Algebra, Ghent University, Krijgslaan 281 Gebouw S22, B 9000 Gent, Belgium. 
of Fourier series of functions to distributions. For example, one of the most basic results in the classical theory says that if $f \in L^{1}[0,2 \pi]$ its (symmetric) Fourier series is $(\mathrm{C}, 1)$ summable at every Lebesgue point $[11,19,46]$, then, this result admits extension. The first extension to periodic distributions was given by G. Walter [42, 43]; moreover, he implicitly obtained the order of Cesàro summability of the symmetric partial sums in terms of the order of the distributional point value. Multidimensional problems for the Fourier transform have been investigated in [12, 38].

A distributional point of view of Fourier series is sometimes more convenient because it provides new interpretations of summability of trigonometric series that the classical point of view hides in somehow. For instance, the relationship between existence of the distributional point value and the Cesàro summability of Fourier series has been achieved in the following result which completely characterizes distributional point values [6]. It is remarkable that such a characterization has not been given for classical functions.

Theorem 1.1. Let $f \in \mathcal{D}^{\prime}(\mathbb{R})$ be a periodic distribution of period $2 \pi$ and let $\sum_{n=-\infty}^{\infty} c_{n} e^{i n x}$ be its Fourier series. Let $x_{0} \in \mathbb{R}$. Then $f\left(x_{0}\right)=\gamma$, in the sense of Eojasiewicz, if and only if there exists $k \in \mathbb{N}$ such that

$$
\lim _{x \rightarrow \infty} \sum_{-x<n \leq a x} c_{n} e^{i n x_{0}}=\gamma \quad(\mathrm{C}, k)
$$

for each $a>0$.

Notice that when $a=1$, the case of symmetric Cesàro means, the above limit reduces to the statement of the $(\mathrm{C}, k)$ summability of the usual sines and cosines Fourier series of the distribution.

We emphasize that the use of asymmetric partial sums is fundamental, since the Cesàro summability of the symmetric partial sums is not enough to conclude the existence of the distributional point value $(f(x)=$ $\sum_{n=1}^{\infty} n \sin n x$ at $x=0$ is an example).

In the case of Fourier integrals of classical functions the situation is similar to that of Fourier series, summability methods must be employed as well. One has also a Cesàro summability version for the Fourier inversion integral formula in a theorem due to Plancherel [27,31]. Other methods of summability are also studied in classical books [3, 4]. Actually, the approach given in $[3,4]$ is very close to distributional point values. Indeed, what they do is to consider pointwise inversion formulas of the type

$$
\lim _{x \rightarrow \infty} \frac{1}{2 \pi} \int_{-\infty}^{\infty} \hat{f}(t) e^{i x_{0} t} \phi\left(\frac{t}{x}\right) \mathrm{d} t=\phi(0) f\left(x_{0}\right),
$$

which is what one usually does in distribution theory when dealing with distributional point values.

In recent works, the authors have been able to characterize the distributional point values of tempered distributions by means of a generalized 
pointwise Fourier inversion formula $[35,36]$. Let us state this result; we will explain the notation later, in Section 2.

Theorem 1.2. Let $f \in \mathcal{S}^{\prime}(\mathbb{R})$. We have $f\left(x_{0}\right)=\gamma$, in the sense of Eojasiewicz, if and only if there exists a $k \in \mathbb{N}$ such that

$$
\frac{1}{2 \pi} \text { e.v. }\left\langle\hat{f}(t), e^{i x_{0} t}\right\rangle=\gamma \quad(\mathrm{C}, k)
$$

which in case $\hat{f}$ is locally integrable means that

$$
\frac{1}{2 \pi} \text { e.v. } \int_{-\infty}^{\infty} \hat{f}(t) e^{i x_{0} t} \mathrm{~d} t=\gamma \quad(\mathrm{C}, k) .
$$

This result includes Theorem 1.1 as a special case and also includes "trigonometric integrals" of tempered functions. Theorem 1.2 provides a complete characterization of the distributional point values of tempered distributions. However, Theorems 1.1 and its extension to tempered distributions, Theorem 1.2, have a little gap, namely, they do not establish a connection between the order of summability of the Fourier inversion formula and the order of the point values (see Section 2 for definitions of these two orders).

Our aim in this work is to establish a relation between these two orders. Among other results, we show that if a tempered distribution, with certain restrictions of growth at $\infty$, has a point value of order $k$, then the special value of the Fourier inversion formula is summable $(\mathrm{C}, k+1)$ to the value. In the case of Fourier series, these restrictions of growth do not appear, hence we generalized the result from [42] and [45]. We also reformulate the result for Fourier series and integrals in terms of summability with respect to a family of kernels, and hence the characterization of distributional point values takes the form of (1.1) for just certain $\phi$ 's taken from this family of summability kernels.

It is interesting to mention that these ideas are closely related to the classical problem of (C) summability for Fourier series (see [46, Chapter XI] and references therein). The first to formulate the problem were Hardy and Littlewood [16, 17]. It basically aims to characterize trigonometric series such that their sines and cosines series,

$$
\frac{a_{0}}{2}+\sum_{n=1}^{\infty}\left(a_{n} \cos n x_{0}+b_{n} \sin n x_{0}\right),
$$

are Cesàro summable at a given point $x_{0}$ and whose coefficients are of slow growth (hence they are tempered distributions!). If we do not care about the order of (C) summability, then distributional point values provide an easy and quick solution to this problem [9, Theorem 6.14.5]. A classical approach to this problem is presented in [46, Chapter XI], where the problem of $(\mathrm{C})$ summability of the symmetric partial sums is investigated with the use of generalized symmetric derivatives of integrated trigonometric series (notion 
which turns out to be equivalent to work with symmetric point values as shown in Section 6).

The paper is organized as follows. In Section 2, we explain the notions and results from the theory of generalized functions (distributions) to be used in this paper. In Section 3, we show that for certain tempered distributions having a point value of order $k$ at a point, the special value of the Fourier inversion formula is summable $(C, \beta)$ to the point value for any $\beta>k$; then, we apply this result to cases of interest; at the end of the section we calculate a bound for the order of summability of the Fourier inversion formula in the general case. Section 4 is devoted to discuss some properties of asymptotically homogeneous functions of degree 0 , which will be a fundamental tool in studying summability of the special value pointwise Fourier inversion formula for tempered distributions. In Section 5, we study the opposite problem, namely, we estimate the order of the point value having the order of Cesàro summability of pointwise Fourier inversion formula. Section 6 is dedicated to the study of symmetric distributional point values; we formulate and solve the problem of symmetric $(\mathrm{C})$ summability for tempered distributions, on the way we recover the classical results for Fourier series $[16,14,45]$. Finally, we study jumps of distributions and find the order in various formulas for the jump originally found in $[36,37]$.

\section{Preliminaries And Notation}

In this section we explain the spaces of test functions and distributions needed in this paper. We also give a summary of the notion of the value of a distribution at a point $[9,21]$, Cesàro behavior and limits of distributions at infinity [7] and some related concepts [35, 36] needed in the future. All of our functions and distributions are over the real line.

The space of test functions $\mathcal{D}$ and $\mathcal{S}$ and the corresponding spaces of distributions $\mathcal{D}^{\prime}$ and $\mathcal{S}^{\prime}$ are well known [29, 41]. Given $\phi \in \mathcal{S}$, we define its Fourier transform by

$$
\mathcal{F}(\phi)(x)=\int_{-\infty}^{\infty} \phi(t) e^{-i x t} \mathrm{~d} t
$$

on $\mathcal{S}^{\prime}$ the Fourier transform is defined as the transpose of the map $\phi \longmapsto$ $\mathcal{F}(\phi)$ from $\mathcal{S}$ to $\mathcal{S}$. We will use indistinctly the notations $\hat{f}, \mathcal{F}(f)$ and $\mathcal{F}\{f(t) ; x\}$ to denote the Fourier transform of $f$.

Denote by $\Gamma$ the Euler gamma function. Recall the definition of the distributions $x_{+}^{\beta} / \Gamma(\beta+1)$, which are defined for all $\beta \in \mathbb{C}$. For $\Re e \beta>$ -1 , they are regular distributions (locally Lebesgue integrable functions) supported on $[0, \infty)$, and they are defined by analytic continuation for the other values of $\beta[9,29,40]$. Note also that when $\beta=-k, k$ being a positive integer, then they reduce to $\delta^{(k)}(x)$, where $\delta(x)$ is the very well known Dirac delta distribution. 
Given a distribution $f \in \mathcal{D}^{\prime}$ supported on the interval $[0, \infty)$, we denote its primitive of order $\beta$ (in short $\beta$-primitive) by the convolution $[40,41]$

$$
f^{(-\beta)}=f * \frac{x_{+}^{\beta-1}}{\Gamma(\beta)} .
$$

Note that the $\beta$-primitive is nothing else but the fractional derivative [41] of order $-\beta$. Since we will frequently use fractional primitives in long calculations, its convenient to introduce some additional notation. Thus, we also denote the $\beta$-primitive by

$$
I_{\beta}\{f(t) ; x\}:=f^{(-\beta)}(x),
$$

so that when $f$ is locally integrable,

$$
I_{\beta}\{f(t) ; x\}=\frac{1}{\Gamma(\beta)} \int_{0}^{x} f(t)(x-t)^{\beta-1} \mathrm{~d} t .
$$

When $f$ is no longer supported on $[0, \infty)$, we cannot in general speak about fractional order primitives. However, if $k \in \mathbb{N}$, we say that $F$ is a $k$-primitive of $f$ if $F^{(k)}=f$. When $f$ is locally integrable (not necessarily supported on $[0, \infty)$ ), we can still use the $k$-primitive given by formula (2.1) with $k=\beta$.

The Cesàro behavior of a distribution at infinity is studied by using the order symbols $O\left(x^{\alpha}\right)$ and $o\left(x^{\alpha}\right)$ in the Cesàro sense [7, 9]. If $f \in \mathcal{D}^{\prime}$ and $\alpha \in \mathbb{R} \backslash\{-1,-2,-3, \ldots\}$, we say that $f(x)=O\left(x^{\alpha}\right)$ as $x \rightarrow \infty$ in the Cesàro sense and write

$$
f(x)=O\left(x^{\alpha}\right) \quad(\mathrm{C}), \quad \text { as } x \rightarrow \infty,
$$

if there exists $k \in \mathbb{N}$ such that every primitive $F$ of order $k$ of $f$ is an ordinary function (locally integrable) for positive large arguments and satisfies the ordinary order relation

$$
F(x)=p(x)+O\left(x^{\alpha+k}\right), \text { as } x \rightarrow \infty,
$$

for a suitable polynomial $p$ of degree at most $k-1$. Note that if $\alpha>-1$, then the polynomial $p$ is irrelevant. To emphasize the order of the Cesàro behavior, it is convenient to write

$$
f(x)=O\left(x^{\alpha}\right) \quad(\mathrm{C}, k), \quad \text { as } x \rightarrow \infty .
$$

A similar definition applies to the little $o$ symbol. The definitions when $x \rightarrow-\infty$ are clear.

Using these ideas, one can define the limit of a distribution at $\infty$ in the Cesàro sense $[7,9]$. We should now extend the definition of limits in the Cesàro sense in order to allow fractional orders.

Definition 2.1. Let $f \in \mathcal{D}^{\prime}$ and $\beta \geq 0$. We say that $f$ has a limit $\ell$ at infinity in the Cesàro sense of order $\beta$ (in the $(\mathrm{C}, \beta)$ sense) and write

$$
\lim _{x \rightarrow \infty} f(x)=\ell \quad(\mathrm{C}, \beta),
$$

if for a decomposition $f=f_{-}+f_{+}$as sum of two distributions supported on $(-\infty, 0]$ and $[0, \infty)$, respectively, one has that the $\beta$-primitive of $f_{+}$is an 
ordinary function (locally integrable) for large arguments and satisfies the ordinary asymptotic relation

$$
f_{+}^{(-\beta)}(x)=\frac{\ell x^{\beta}}{\Gamma(\beta+1)}+o\left(x^{\beta}\right), \text { as } x \rightarrow \infty .
$$

The reader can easily check that the definition does not depend on the decomposition $f=f_{-}+f_{+}$. If we do not want to make reference to the order $\beta$ in (2.2), we simply write

$$
\lim _{x \rightarrow \infty} f(x)=\ell \quad(\mathrm{C}) .
$$

We now turn our attention to the local behavior of generalized functions $[9,26,39,40]$. Łojasiewicz [21] defined the value of a distribution $f \in \mathcal{D}^{\prime}$ at the point $x_{0}$ as the limit

$$
f\left(x_{0}\right):=\gamma=\lim _{\varepsilon \rightarrow 0} f\left(x_{0}+\varepsilon x\right),
$$

if the limit exists in $\mathcal{D}^{\prime}$, that is if

$$
\lim _{\varepsilon \rightarrow 0}\left\langle f\left(x_{0}+\varepsilon x\right), \phi(x)\right\rangle=\gamma \int_{-\infty}^{\infty} \phi(x) \mathrm{d} x
$$

for each $\phi \in \mathcal{D}$. Since (2.3) is only supposed to hold for $\phi \in \mathcal{D}$, we emphasize this fact saying that $f\left(x_{0}\right)=\gamma$ in $\mathcal{D}^{\prime}$, in case that (2.3) is satisfied. Suppose now that $f \in \mathcal{S}^{\prime}$ and $f\left(x_{0}\right)=\gamma$ in $\mathcal{D}^{\prime}$; initially, (2.3) does not have to be true for $\phi \in \mathcal{S}$. However, it is shown in [8, Corollary 1] and [39, Section 6] that if (2.3) holds for $\phi \in \mathcal{D}$, it will remain true for $\phi \in \mathcal{S}$; so we are in the right to say $f\left(x_{0}\right)=\gamma$ in $\mathcal{S}^{\prime}$, and this is equivalent to the existence of $f\left(x_{0}\right)$ in $\mathcal{D}^{\prime}$.

It was shown by Łojasiewicz [21] that the existence of the distributional point value $f\left(x_{0}\right)=\gamma$ is equivalent to the existence of $n \in \mathbb{N}$, and a primitive of order $n$ of $f$, that is $F^{(n)}=f$, which is continuous in a neighborhood of $x_{0}$ and satisfies

$$
\lim _{x \rightarrow x_{0}} \frac{n ! F(x)}{\left(x-x_{0}\right)^{n}}=\gamma .
$$

Note that (2.4) says that any $n$-primitive of $f$ has an $n^{\text {th }}$ differential in the sense of Peano [2] (see also [46, Chapter XI, page 59] in connection with differentiated trigonometric series).

It is convenient to define the order of the value of a tempered distribution at a point. Our definition differs from that of Eojasiewicz [21]. We should adopt a little variant of Łojasiewicz's original definition for the sake of convenience.

Definition 2.2. Let $f$ be a tempered distribution. We say that $f$ has a (distributional) point value $\gamma$ at $x_{0}$ in $\mathcal{S}^{\prime}$ of order $n$, and write $f\left(x_{0}\right)=\gamma$ in $\mathcal{S}^{\prime}$ with order $n$, if $n$ is the minimum integer such that there exists a locally bounded measurable function $F$ of polynomial growth at infinity such that $F^{(n)}=f$ and $F$ satisfies (2.4). 
A similar definition has been also adopted in [44, Section 8.3, Definition 8.1] for studying distributional point values of tempered distributions in relation with orthogonal wavelets and multiresolution analysis approximations for spaces of tempered distributions.

The final concept we would like to discuss here is that of distributional evaluations in the e.v. Cesàro sense, as defined by the authors in [35, 36].

Definition 2.3. Let $g \in \mathcal{D}^{\prime}, \phi \in C^{\infty}(\mathbb{R})$ and $\beta \geq 0$. We say that the evaluation $\langle g(x), \phi(x)\rangle$ exists in the e.v. Cesàro sense (of order $\beta$ ), and write

$$
\text { e.v. }\langle g(x), \phi(x)\rangle=\gamma \quad(\mathrm{C}, \beta),
$$

if for some primitive $G$ of $g \phi$ and $\forall a>0$ we have

$$
\lim _{x \rightarrow \infty}(G(a x)-G(-x))=\gamma \quad(\mathrm{C}, \beta) .
$$

If $g$ is locally integrable then we write $(2.5)$ as

$$
\text { e.v. } \int_{-\infty}^{\infty} g(x) \phi(x) \mathrm{d} x=\gamma \quad(\mathrm{C}, \beta) .
$$

If (2.5) exists, we also say that the special value of the evaluation exists in the $(\mathrm{C}, \beta)$ sense. Similarly for $(2.6)$, we say that the special value of the integral exists in the $(\mathrm{C}, \beta)$ sense.

The last definition allows us to make sense out of the Fourier inversion formula; indeed $[35,36]$, the authors have shown that $f \in \mathcal{S}^{\prime}$ has a point value $\gamma$ at $x_{0}$ if and only if

$$
\text { e.v. }\left\langle\hat{f}(x), e^{i x_{0} x}\right\rangle=2 \pi \gamma \quad(\mathrm{C}, k),
$$

for some $k \in \mathbb{N}$. As we mentioned at the Introduction, this result does not say anything about the relationship between the order of summability of this inversion formula and the order of the point value; this will be the main subject of Section 3 and Section 5 in the present article.

\section{ORDER OF SUMMABILITY}

In this section we obtain a bound for the order of summability of the Fourier inversion formula for tempered distributions in the general case. We also analyze two particular cases, the case of Fourier series and the case of distributions with compact support; in both cases we obtain the expected result: if the distribution has a value of order $k$, then the order of summability of the Fourier inversion formula is at least $k+1$.

Suppose that $f \in \mathcal{S}^{\prime}$ is so that $\hat{f} \in L_{\text {loc }}^{1}(\mathbb{R})$. Denote by $\theta_{A}$ the characteristic function of a set $A$. Then note that (2.7) holds if and only if

$$
\lim _{x \rightarrow \infty} \frac{1}{2 \pi} \int_{-\infty}^{\infty} \hat{f}(t) e^{i x_{0} t} \phi_{a}^{\beta}\left(\frac{t}{x}\right) \mathrm{d} t=f\left(x_{0}\right),
$$


where $\phi_{a}^{\beta}$ is the summability kernel given by

$$
\phi_{a}^{\beta}(t)=(1+t)^{\beta} \theta_{[-1,0]}(t)+\left(1-\frac{t}{a}\right)^{\beta} \theta_{[0, a]}(t) .
$$

Indeed, this follows directly from Definition 2.3. Observe that we may consider (3.2) as the summability kernels of asymmetric $(\mathrm{C}, \beta)$ means. Notice also that if (3.1) holds for some $\beta$, then it holds for any $\tilde{\beta} \geq \beta$. We shall need some properties of these kernels, they are stated in the next lemma.

Lemma 3.1. Suppose that $0<\beta \leq 1$. Then,

$$
\left|\hat{\phi}_{a}^{\beta}(t)\right| \leq \frac{2+3 \beta\left(1+a^{-1}\right)}{t^{\beta+1}} \quad, t>1 .
$$

Moreover, $\int_{-\infty}^{\infty} \hat{\phi}_{a}^{\beta}(t) \mathrm{d} t=2 \pi$.

Proof. Suppose the inequality is satisfied, then $\hat{\phi}_{a}^{\beta} \in L^{1}(\mathbb{R}) \cap L^{2}(\mathbb{R})$, so the very well known classical result [4, page 62$]$ implies that the pointwise Fourier inversion formula holds in this case, and thus we have $\int_{-\infty}^{\infty} \hat{\phi}_{a}^{\beta}(t) \mathrm{d} t=$ $2 \pi \phi_{a}^{\beta}(0)=2 \pi$. Let us now show the inequality.

$$
\begin{aligned}
\left|\hat{\phi}_{a}^{\beta}(t)\right| & =\left|\int_{0}^{1}(1-u)^{\beta}\left(e^{i t u}+a e^{-i a t u}\right) \mathrm{d} u\right| \\
& =\frac{\beta}{t}\left|\int_{0}^{1}(1-u)^{\beta-1}\left(e^{-i a t u}-e^{i t u}\right) \mathrm{d} u\right| \\
& =\frac{\beta}{t^{\beta+1}}\left|\int_{0}^{t} u^{\beta-1}\left(e^{-i a t} e^{i a u}-e^{i t} e^{-i u}\right) \mathrm{d} u\right| \\
& \leq \frac{2}{t^{\beta+1}}+\frac{\beta}{t^{\beta+1}}\left|\int_{1}^{t} u^{\beta-1}\left(e^{-i a t} e^{i a u}-e^{i t} e^{-i u}\right) \mathrm{d} u\right| \\
& \leq \frac{2}{t^{\beta+1}}+\frac{\beta}{t^{\beta+1}}\left(a^{-1}+1\right)\left(1+t^{\beta-1}+(1-\beta) \int_{1}^{\infty} u^{\beta-2} \mathrm{~d} u\right),
\end{aligned}
$$

where in the last step we have used integration by parts.

The explicit value of the constant term in the bound from the last lemma is unimportant, however, we will use the fact that this estimate holds uniformly for $a$ on compact subsets of $(0, \infty)$.

We start to study the pointwise Fourier inversion formula. We first show a proposition concerning the $L^{2}(\mathbb{R})$-case. The proof of the following proposition is similar to that of [31, Theorem 13], but we include it for the sake of completeness.

Proposition 3.2. Suppose that $g \in L^{2}(\mathbb{R})$. If $g$ is continuous at $x_{0}$, then we have for any $\beta>0$,

$$
\frac{1}{2 \pi} \text { e.v. }\left\langle\hat{g}(t), e^{i x_{0} t}\right\rangle=g\left(x_{0}\right) \quad(\mathrm{C}, \beta),
$$


or, which amounts to the same,

$$
\lim _{x \rightarrow \infty} \frac{1}{2 \pi} \int_{-\infty}^{\infty} \hat{g}(t) e^{i x_{0} t} \phi_{a}^{\beta}\left(\frac{t}{x}\right) \mathrm{d} t=g\left(x_{0}\right),
$$

uniformly for a on compact subsets of $(0, \infty)$.

Proof. By considering $g\left(x+x_{0}\right)$, we may assume that $x_{0}=0$. We may also assume that $0<\beta \leq 1$, because if it holds for those values of $\beta$, then it holds for any $\beta>0$.

We have that

$$
\int_{-\infty}^{\infty} \hat{g}(t) \phi_{a}^{\beta}\left(\frac{t}{x}\right) \mathrm{d} t=x \int_{-\infty}^{\infty} g(t) \hat{\phi}_{a}^{\beta}(x t) \mathrm{d} t .
$$

Therefore (3.3) holds if and only if

$$
\lim _{x \rightarrow \infty} \int_{-\infty}^{\infty} g(t) K_{a}^{\beta}(t, x) \mathrm{d} t=g(0),
$$

where $K_{a}^{\beta}(t, x)=x \hat{\phi}_{a}^{\beta}(x t) /(2 \pi)$. Now, because of Lemma 3.1 and the boundedness of $\hat{\phi}_{a}^{\beta}$, the kernel $K_{a}^{\beta}(t, x)$ satisfies the following properties

$$
\int_{-\infty}^{\infty} K_{a}^{\beta}(t, x) d t=1,\left|K_{a}^{\beta}(t, x)\right| \leq M x,\left|K_{a}^{\beta}(t, x)\right| \leq \frac{N}{x^{\beta} t^{\beta+1}},
$$

for some positives constants $M$ and $N$, and the last inequality being valid for $x|t| \geq 1$. The estimates are satisfied uniformly for $a$ on compact sets. Pick $\delta>0$ such that if $|t|<\delta$ then $|g(t)-g(0)|<\varepsilon$; keep $x^{-1}<\min \{\varepsilon, \delta\}$, then

$$
\begin{aligned}
& \left|\int_{-\infty}^{\infty}(g(t)-g(0)) K_{a}^{\beta}(t, x) \mathrm{d} t\right| \\
& \leq \varepsilon \int_{-\frac{1}{x}}^{\frac{1}{x}}\left|K_{a}^{\beta}(t, x)\right| \mathrm{d} t+\int_{|t| \geq \frac{1}{x}}|g(t)-g(0)|\left|K_{a}^{\beta}(t, x)\right| \mathrm{d} t \\
& \leq 2 \varepsilon\left(M+N \beta^{-1}\right)+\frac{N}{x^{\beta}} \int_{|t| \geq \delta}^{\infty} \frac{|g(t)-g(0)|}{t^{\beta+1}} \mathrm{~d} t
\end{aligned}
$$

hence,

$$
\varlimsup_{x \rightarrow \infty}\left|\frac{1}{2 \pi} \int_{-\infty}^{\infty} \hat{g}(t) \phi_{a}\left(\frac{t}{x}\right) \mathrm{d} t-g(0)\right| \leq 2 \varepsilon\left(M+N \beta^{-1}\right),
$$

since $\varepsilon$ is arbitrary, this completes the proof.

Remark 3.3. Proposition 3.2 still holds if one assumes that $x_{0}$ is a Lebesgue point of $g$ instead of the continuity at $x_{0}$. This proposition is also true for kernels $\phi$ other than $\phi_{a}^{\beta}$; in fact, the proposition is valid if $K(t, x)=x \phi(x t) /(2 \pi)$ satisfies (3.4), that is $K(t, x)$ satisfies $\int_{-\infty}^{\infty} K(t, x) \mathrm{d} t=1,|K(t, x)| \leq M x$ for $|t| x<B$ and $|K(t, x)|<N x^{-\alpha} t^{-\alpha-1}$, for some positive constants $B, M, N$ and $\alpha$. For other related results, the reader can consult Titchmarsh's book [31, Chapter 1]. 
In order to make further progress, we need two formulas. They are stated in the next two lemmas.

Lemma 3.4. Let $h \in \mathcal{D}^{\prime}$ and $m, k \in \mathbb{N}$. Suppose that $m \geq k$, then

$$
x^{k} h^{(m)}(x)=\sum_{j=0}^{k}(-1)^{j} \frac{k !}{(k-j) !}\left(\begin{array}{c}
m \\
j
\end{array}\right) \frac{d^{m-j}}{d x^{m-j}}\left(x^{k-j} h(x)\right) \text {. }
$$

Proof. It follows directly form the very well known formula [5, Lemma 1.3], valid if $\varphi \in C^{\infty}(\mathbb{R})$,

$$
\varphi h^{(m)}=\sum_{j=0}^{m}(-1)^{j}\left(\begin{array}{c}
m \\
j
\end{array}\right) \frac{d^{m-j}}{d x^{m-j}}\left(\varphi^{(j)} h\right)
$$

applied to $\varphi(x)=x^{k}$.

Lemma 3.5. Let $h$ be a locally integrable function supported on $[0, \infty)$. For any positive number $\beta$ and positive integer $k$

$$
I_{\beta}\left\{t^{k} h(t) ; x\right\}=\sum_{j=0}^{k}(-1)^{j}\left(\begin{array}{l}
k \\
j
\end{array}\right) \frac{\Gamma(\beta+j)}{\Gamma(\beta)} x^{k-j} h^{(-\beta-j)}(x) .
$$

Proof. We proceed by induction over $k$. For $k=1$,

$$
\begin{aligned}
I_{\beta}\{t h(t) ; x\} & =\frac{1}{\Gamma(\beta)} \int_{0}^{x}(x-t)^{\beta-1} t h(t) \mathrm{d} t \\
& =x h^{(-\beta)}(x)-\frac{1}{\Gamma(\beta)} \int_{0}^{x}\left(\int_{0}^{t}(x-u)^{\beta-1} h(u) \mathrm{d} u\right) \mathrm{d} t \\
& =x h^{(-\beta)}(x)-\frac{1}{\Gamma(\beta)} \int_{0}^{x}(x-u)^{\beta} h(u) \mathrm{d} u \\
& =x h^{(-\beta)}(x)-\beta h^{(-\beta-1)}(x) .
\end{aligned}
$$

If the formula is true for $k$, then

$$
\begin{aligned}
I_{\beta}\left\{t^{k+1} h(t) ; x\right\}= & \sum_{j=0}^{k}(-1)^{j}\left(\begin{array}{c}
k \\
j
\end{array}\right) \frac{\Gamma(\beta+j)}{\Gamma(\beta)} x^{k-j} I_{\beta+j}\{t h(t) ; x\} \\
= & \sum_{j=0}^{k}(-1)^{j}\left(\begin{array}{c}
k \\
j
\end{array}\right) \frac{\Gamma(\beta+j)}{\Gamma(\beta)} x^{k+1-j} h^{(-\beta-j)}(x) \\
& -\sum_{j=0}^{k}(-1)^{j}\left(\begin{array}{c}
k \\
j
\end{array}\right) \frac{\Gamma(\beta+j)}{\Gamma(\beta)}(\beta+j) x^{k-j} h^{(-\beta-j-1)}(x) \\
= & \sum_{j=0}^{k+1}(-1)^{j}\left(\begin{array}{c}
k+1 \\
j
\end{array}\right) \frac{\Gamma(\beta+j)}{\Gamma(\beta)} x^{k+1-j} h^{(-\beta-j)}(x) .
\end{aligned}
$$


We begin to analyze the case of tempered distributions, by first imposing some strong restrictions to the behavior of the distribution at infinity.

Theorem 3.6. Let $f \in \mathcal{S}^{\prime}$. Suppose that there exists an $m \in \mathbb{N}$ such that every $m$-primitive of $f$ is a locally integrable function for large arguments and satisfies an estimate $O\left(|x|^{m-1}\right)$, as $x \rightarrow \infty$. If $f$ has a distributional point value $f\left(x_{0}\right)=\gamma$ at $x_{0}$ in $\mathcal{S}^{\prime}$, whose order is $n$, then

$$
\frac{1}{2 \pi} \mathrm{e} . \mathrm{v} \cdot\left\langle\hat{f}(x), e^{i x_{0} x}\right\rangle=\gamma \quad(\mathrm{C}, \beta)
$$

for any $\beta>k=\max \{m, n\}$.

Proof. We can assume that $x_{0}=0$. Take $h$, a $k$-primitive of $f$, such that $h$ is a locally bounded measurable function and $h(x)=O\left(|x|^{k-1}\right)$, as $|x| \rightarrow \infty$, and $h(x)=\gamma x^{k} / k !+o\left(|x|^{k}\right)$ as $x \rightarrow 0$. Set $g(x)=h(x) / x^{k}$, then $g \in L^{2}(\mathbb{R})$ and $g$ is continuous at 0 with $g(0)=\gamma / k$ ! . Consider $\hat{g} \in L^{2}(\mathbb{R})$. Then,

$$
(\hat{g})^{(k)}(x)=(-i)^{k} \mathcal{F}\left\{t^{k} g(t) ; x\right\}=(-i)^{k} \mathcal{F}\{h(t) ; x\}=(-i)^{k} \hat{h}(x) .
$$

Thus,

$$
\hat{f}(x)=\mathcal{F}\left\{h^{(k)}(t) ; x\right\}=i^{k} x^{k} \hat{h}(x)=(-1)^{k} x^{k}(\hat{g})^{(k)}(x) .
$$

We now look at a $k$-primitive of $\hat{f}$. Indeed, by (3.5) and Lemma 3.4

$$
F(x)=\sum_{j=0}^{k}(-1)^{k-j} \frac{k !}{(k-j) !}\left(\begin{array}{c}
k \\
j
\end{array}\right) I_{j}\left\{t^{k-j} \hat{g}(t)(t) ; x\right\}
$$

is a $k$-primitive of $\hat{f}$. Let $\beta>k$ and $a>0$. Set $\tilde{\beta}=\beta-k$. To show the theorem, one should prove that

$$
F_{1}(x):=\frac{1}{a^{k-1}} F(a x)+(-1)^{k} F(-x)=\frac{2 \pi \gamma x^{k-1}}{(k-1) !}+o\left(x^{k-1}\right) \quad(\mathrm{C}, \beta-k+1)
$$

as $x \rightarrow \infty$. Therefore, we have to show that

$$
\begin{aligned}
I_{\tilde{\beta}+1}\left\{F_{1}(t) ; x\right\} & =\frac{1}{\Gamma(\tilde{\beta}+1)} \int_{0}^{x}(x-t)^{\tilde{\beta}} F_{1}(t) \mathrm{d} t \\
& =\frac{2 \pi \gamma x^{\beta}}{\Gamma(\beta+1)}+o\left(x^{\beta}\right), \text { as } x \rightarrow \infty .
\end{aligned}
$$

Notice that

$$
\begin{aligned}
& a^{1-k} I_{j}\left\{t^{k-j} \hat{g}(t) ; a x\right\}+(-1)^{k} I_{j}\left\{t^{k-j} \hat{g}(a t) ;-x\right\} \\
& =I_{j}\left\{t^{k-j}(a \hat{g}(t)+\hat{g}(-t)) ; x\right\}
\end{aligned}
$$


So, setting $g_{1}(t):=a \hat{g}(a t)+\hat{g}(-t)$ for $t \geq 0$ and $g_{1}(t):=0$ for $t<0$ we obtain from Lemma 3.4 and (3.6)

$$
\begin{aligned}
F_{1}(x) & =\sum_{j=0}^{k}(-1)^{k-j} \frac{k !}{(k-j) !}\left(\begin{array}{c}
k \\
j
\end{array}\right) I_{j}\left\{t^{k-j} g_{1}(t) ; x\right\} \\
& =(-1)^{k} I_{k}\left\{t^{k} g_{1}^{(k)}(t) ; x\right\}, \quad \text { for } x>0,
\end{aligned}
$$

then, by Lemma 3.5, for $x>0$

$$
\begin{aligned}
I_{\tilde{\beta}+1}\left\{F_{1}(t) ; x\right\} & =(-1)^{k} I_{\beta+1}\left\{t^{k} g_{1}^{(k)}(t) ; x\right\} \\
& =\sum_{j=0}^{k}(-1)^{k-j}\left(\begin{array}{c}
k \\
j
\end{array}\right) \frac{\Gamma(\beta+1+j)}{\Gamma(\beta+1)} x^{k-j} g_{1}^{(-\tilde{\beta}-1-j)}(x),
\end{aligned}
$$

but

$$
g_{1}^{(-\tilde{\beta}-1-j)}(x)=\frac{x^{\tilde{\beta}+j}}{\Gamma(\tilde{\beta}+1+j)} \int_{-\infty}^{\infty} \hat{g}(t) \phi_{a}^{\tilde{\beta}+j}\left(\frac{t}{x}\right) \mathrm{d} t \sim \frac{2 \pi \gamma x^{\tilde{\beta}+j}}{k ! \Gamma(\tilde{\beta}+1+j)},
$$

as $x \rightarrow \infty$, where the last asymptotic relation holds in view of Proposition 3.2 , the continuity of $g$ at 0 , and the fact $g(0)=\gamma / k$ !. Therefore,

$$
\begin{aligned}
I_{\tilde{\beta}+1}\left\{F_{1}(t) ; x\right\} & =\frac{2 \pi \gamma x^{\beta}}{k ! \Gamma(\beta+1)} \sum_{j=0}^{k}(-1)^{k-j}\left(\begin{array}{c}
k \\
j
\end{array}\right) \frac{\Gamma(\beta+1+j)}{\Gamma(\beta-k+1+j)}+o\left(x^{\beta}\right) \\
& =\left.\frac{2 \pi \gamma x^{\beta}}{k ! \Gamma(\beta+1)} \sum_{j=0}^{k}\left(\begin{array}{c}
k \\
j
\end{array}\right)(-1)^{k-j} \frac{d^{k}}{d t^{k}}\left(t^{\beta+j}\right)\right|_{t=1}+o\left(x^{\beta}\right) \\
& =\left.\frac{2 \pi \gamma x^{\beta}}{k ! \Gamma(\beta+1)} \frac{d^{k}}{d t^{k}}\left(t^{\beta} \sum_{j=0}^{k}\left(\begin{array}{l}
k \\
j
\end{array}\right)(-1)^{k-j} t^{j}\right)\right|_{t=1}+o\left(x^{\beta}\right) \\
& =\frac{2 \pi \gamma x^{\beta}}{\Gamma(\beta+1)}\left(\left.\frac{1}{k !} \frac{d^{k}}{d t^{k}}\left(t^{\beta}(t-1)^{k}\right)\right|_{t=1}\right)+o\left(x^{\beta}\right) \\
& =\frac{2 \pi \gamma x^{\beta}}{\Gamma(\beta+1)}+o\left(x^{\beta}\right) \quad \text { as } x \rightarrow \infty ;
\end{aligned}
$$

hence, we have established (3.7), as required.

Remark 3.7. It follows from the proof of the last theorem and Proposition 3.2 that (3.7) holds uniformly for $a$ in compact subsets of $(0, \infty)$.

The next corollary follows directly from equation (3.6).

Corollary 3.8. Under the hypothesis of Theorem 3.6, then $\hat{f}$ is the $k^{\text {th }}$ derivative of a locally integrable function. 
Although it imposes conditions on the behavior at infinity of the tempered distribution, we may apply Theorem 3.6 to several cases of special interest. The next two corollaries follow directly from Theorem 3.6 (for the direct application of Theorem 3.6 in Corollary 3.10 one should argue that it is enough to assume $c_{0}=0$ ).

Corollary 3.9. Let $f$ be a distribution with compact support. Suppose that $f\left(x_{0}\right)=\gamma$ in $\mathcal{S}^{\prime}$ with order $k$. Then for each $a>0$ and $\beta>k$,

$$
\lim _{x \rightarrow \infty} \frac{1}{2 \pi} \int_{-x}^{a x} \hat{f}(t) e^{i x_{0} t} \mathrm{~d} t=\gamma \quad(\mathrm{C}, \beta),
$$

or which is the same

$$
\lim _{x \rightarrow \infty} \frac{1}{2 \pi} \int_{-\infty}^{\infty} \phi_{a}^{\beta}\left(\frac{t}{x}\right) \hat{f}(t) e^{i x_{0} t} \mathrm{~d} t=\gamma .
$$

Moreover, relation (3.8) holds uniformly for a in compact subsets of $(0, \infty)$.

Corollary 3.10. Let $f$ be a $2 \pi$-periodic distribution, with Fourier series $\sum_{n=-\infty}^{\infty} c_{n} e^{i x n}$. Suppose that $f\left(x_{0}\right)=\gamma$ in $\mathcal{S}^{\prime}$ with order $k \geq 1$. Then for each $a>0$ and $\beta>k$,

$$
\lim _{x \rightarrow \infty} \sum_{-x \leq n \leq a x} c_{n} e^{i x_{0} n}=\gamma \quad(\mathrm{C}, \beta),
$$

or equivalently

$$
\lim _{x \rightarrow \infty} \sum_{-x \leq n \leq a x} \phi_{a}^{\beta}\left(\frac{n}{x}\right) c_{n} e^{i x_{0} n}=\gamma .
$$

Moreover, relation (3.9) holds uniformly for a in compact subsets of $(0, \infty)$.

As a particular case of Corollary 3.10, we obtain almost everywhere summability of order $\beta>1$ for Denjoy integrable functions [13, 20]. This result extends that of Privalov (see [20, page 573]) which only considers the symmetric series. The reader should notice that Privalov theorem is included in the much stronger result of Marcinkiewicz [23], [46, Chapter XI, Theorem 5.4].

Corollary 3.11. Let $f$ be a $2 \pi$-periodic function which is Denjoy integrable on $[-\pi, \pi]$. Let $\beta>1$. If its Fourier series is $\sum_{n=-\infty}^{\infty} c_{n} e^{i x n}$, then we have for almost every $x_{0}$

$$
\lim _{x \rightarrow \infty} \sum_{-x \leq n \leq a x} \phi_{a}^{\beta}\left(\frac{n}{x}\right) c_{n} e^{i x_{0} n}=f\left(x_{0}\right), \quad \text { for all } a>0 .
$$

We now aboard the case of general behavior at infinity. For that, we need the following two lemmas.

Lemma 3.12. Let $g \in L^{2}(\mathbb{R})$. Suppose that $x_{0} \notin \operatorname{supp} g$, then,

$$
\lim _{x \rightarrow \infty} \int_{-x}^{a x} \hat{g}(t) e^{i x_{0} t} \mathrm{~d} t=0,
$$

uniformly for a in compact subsets of $(0, \infty)$. 
Proof. The proof is trivial, just apply Parseval's relation and then use RiemannLebesgue lemma.

Lemma 3.13. Let $f \in \mathcal{S}^{\prime}$. Suppose that $x_{0} \notin \operatorname{supp} f$ and that

$$
f(x)=O\left(|x|^{\alpha}\right) \quad(\mathrm{C}), \text { as }|x| \rightarrow \infty,
$$

for some $\alpha>-1$. Let $m$ be the minimum integer such that any m-primitive of $f$ is locally bounded and $O\left(|x|^{m+\alpha}\right)$ as $|x| \rightarrow \infty$. Then

$$
\text { e.v. }\left\langle f(x), e^{i x_{0} x}\right\rangle=0 \quad(\mathrm{C}, k),
$$

where $k=\left[m+\alpha+\frac{1}{2}\right]+1$ ( [ $\left.\cdot\right]$ stands for the integral part of a number $)$.

Proof. The proof is completely analogous to that of Theorem 3.6. We may assume that $x_{0}=0$. Let $h$ be an $m$-primitive of $f$ such that $h$ is 0 in a neighborhood of 0 and

$$
h(x)=O\left(|x|^{m+\alpha}\right) \quad \text { as }|x| \rightarrow \infty .
$$

Set $g(x)=h(x) / x^{k}$, then $g$ satisfies the hypothesis of Lemma 3.12. Define $G(x)=\int_{0}^{x} \hat{g}(t) d t$; by Lemma 3.4, the following function is a $(k+1)$-primitive of $\hat{f}$

$$
F(x)=\sum_{j=0}^{k}(-1)^{k-j} \frac{k !}{(k-j) !}\left(\begin{array}{c}
k+1 \\
j
\end{array}\right) I_{j}\left\{t^{k-j} G(t) ; x\right\}
$$

Since

$\frac{1}{a^{k}} I_{j}\left\{t^{k-j} G(t) ; a x\right\}-(-1)^{k} I_{j}\left\{t^{k-j} G(t) ;-x\right\}=I_{j}\left\{t^{k-j} \int_{-t}^{a t} \hat{g}(u) \mathrm{d} u ; x\right\}$,

we can use Lemma 3.12 to conclude

$$
\begin{aligned}
\frac{1}{a^{k}} F(x)-(-1)^{k} F(-x) & =\sum_{j=0}^{k}(-1)^{k-j} \frac{k !}{(k-j) !}\left(\begin{array}{c}
k+1 \\
j
\end{array}\right) I_{j}\left\{o\left(t^{k-j}\right) ; x\right\} \\
& =o\left(x^{k}\right) \quad \text { as } x \rightarrow \infty,
\end{aligned}
$$

uniformly for $a$ on compact subsets of $(0, \infty)$.

We now combine Theorem 3.6 and and Lemma 3.13 to obtain a bound for the order of summability of the Fourier inversion formula of a general tempered distribution. We remark that every tempered distribution satisfies an estimate of type (3.10).

Theorem 3.14. Let $f \in \mathcal{S}^{\prime}$ have the behavior at infinity

$$
f(x)=O\left(|x|^{\alpha}\right) \quad(\mathrm{C}, m), \text { as }|x| \rightarrow \infty .
$$

If $f\left(x_{0}\right)=\gamma$ in $\mathcal{S}^{\prime}$ with order $n$, then

$$
\frac{1}{2 \pi} \mathrm{e} \cdot \mathrm{v} \cdot\left\langle\hat{f}(x), e^{i x_{0} x}\right\rangle=\gamma \quad(\mathrm{C}, k+1),
$$

where $k=\max \left\{m, n,\left[n+\alpha+\frac{1}{2}\right],\left[m+\alpha+\frac{1}{2}\right]\right\}$. 


\section{Asymptotically Homogeneous Functions}

In this section we discuss some properties of asymptotically homogeneous functions of degree 0 , they are a fundamental tool in the study of summability of distributional evaluations in the e.v. Cesàro sense $[6,9,36]$. They have also shown to be important in the study of quasiasymptotics of distributions $[33,34,39]$. We will apply these properties in the next section.

We say that a measurable function $\sigma$, defined in an interval of the form $[A, \infty) \subset(0, \infty)$, is asymptotically homogeneous of degree 0 if for each $a>0$, we have

$$
\sigma(a x)=\sigma(x)+o(1) \text { as } x \rightarrow \infty .
$$

Most of the properties discussed here are already known [9]; however, we will take a different approach to them, which is based on the concept of slowly varying functions in the sense of Karamata [30]. Suppose $\sigma$ satisfies (4.1) for each $a>0$, we may assume that $\sigma$ is real valued, otherwise we consider its real and imaginary parts separately. Then $L(x)=e^{\sigma(x)}$ is positive and measurable on $[A, \infty)$ and for each $a>0$

$$
\lim _{x \rightarrow \infty} \frac{L(a x)}{L(x)}=1
$$

Therefore, $L$ is a slowly varying function. It is very well known that (4.2) must hold uniformly for $a$ in compact subsets of $(0, \infty)$ [30], so should (4.1). Actually, if one only assumes that (4.1) holds in a set of positive measure, then it holds for every $a>0$; we will use this property implicitly sometimes in the future. In addition, there is a very well known representation formula for slowly varying functions [30, Theorem 1.2], which implies that there exists a number $B \geq A$ such that

$$
\sigma(x)=\eta(x)+\int_{B}^{x} \frac{\tau(t)}{t} \mathrm{~d} t, \quad x \geq B,
$$

where $\eta$ is a bounded measurable function such that $\eta(x) \rightarrow c$ as $x \rightarrow \infty$ $(|c|<\infty)$, and $\tau$ is a continuous function on $[B, \infty)$ such that $\tau(x) \rightarrow 0$ as $x \rightarrow \infty$. Relation (4.3) implies two estimates for the growth of $\sigma$, first,

$$
\sigma(x)=o(\log x), \quad \text { as } x \rightarrow \infty ;
$$

secondly, there are two constants $A_{0}$ and $A_{1}$ such that

$$
|\sigma(a x)-\sigma(x)| \leq A_{0}|\log a|+A_{1},
$$

for $x \geq B$ and $a x \geq B$. The last inequality implies the following lemma.

Lemma 4.1. Let $\sigma$ be an asymptotically homogeneous function of degree 0 defined on $(0, \infty)$. Let $g$ be a function such that $g(t)(1+|\log t|)$ is in $L^{1}(0, \infty)$. Suppose that at least one of the following two condition is satisfied:

i) $\sigma$ is bounded in each finite subinterval of $(0, \infty)$

ii) $\sigma \in L_{\mathrm{loc}}^{1}([0, \infty))$ and $g$ is bounded near the origin 
then we have that

$$
\int_{0}^{\infty} \sigma(x t) g(t) \mathrm{d} t=\sigma(x) \int_{0}^{\infty} g(t) \mathrm{d} t+o(1), \quad \text { as } x \rightarrow \infty .
$$

Proof. Choose $B$ as in (4.3), we keep $x>B$. Consider

$$
\int_{0}^{\infty}(\sigma(x t)-\sigma(x)) g(t) \mathrm{d} t=J_{1}(x)+J_{2}(x)-J_{3}(x),
$$

where $J_{1}(x)=\int_{B / x}^{\infty}(\sigma(x t)-\sigma(x)) g(t) \mathrm{d} t, J_{2}(x)=\int_{0}^{B / x} \sigma(x t) g(t) \mathrm{d} t$, and $J_{3}(x)=\sigma(x) \int_{0}^{B / x} g(t) \mathrm{d} t$. Because of (4.5) and the assumption over $g$, we can apply Lebesgue Dominated Convergence Theorem to conclude that $J_{1}(x)=o(1)$ as $x \rightarrow \infty$. That $J_{2}(x)=o(1)$ as $x \rightarrow \infty$ follows easily from the assumptions. Finally, by using (4.4), we obtain that

$$
\left|J_{3}(x)\right| \leq \frac{|\sigma(x)|}{\log x+1-\ln B} \int_{0}^{\infty}(1+|\log t|)|g(t)| \mathrm{d} t=o(1), \quad \text { as } x \rightarrow \infty .
$$

\section{ORder of POINT VAlue}

In this section we show that if e.v. $\left\langle\hat{f}(x), e^{i x x_{0}}\right\rangle=2 \pi \gamma(\mathrm{C}, k)$, then $f\left(x_{0}\right)=\gamma$ in $\mathcal{D}^{\prime}$ and the order of the point value is less or equal to $k+2$.

We begin with a particular case which has its inspiration in Riemann's theorems on the formal integration of trigonometrical series [46, Chapter IX, page 319].

Theorem 5.1. Let $f$ be an element of $\mathcal{S}^{\prime}$. Suppose that

$$
\frac{1}{2 \pi} \text { e.v. }\left\langle\hat{f}(x), e^{i x_{0} x}\right\rangle=\gamma \quad(\mathrm{C}, 0),
$$

then, $f\left(x_{0}\right)=\gamma$ in $\mathcal{S}^{\prime}$; moreover if $F_{1}$ and $F_{2}$ are any primitives of order 1 and 2 respectively, then $F_{1}$ is locally integrable and $F_{2}$ possesses a Peano second order differential at $x_{0}$, with $\gamma$ as the second order term, i.e., $F_{2}$ is differentiable at $x_{0}$ and as $x \rightarrow x_{0}$

$$
F_{2}(x)=F_{2}\left(x_{0}\right)+F_{2}^{\prime}\left(x_{0}\right)\left(x-x_{0}\right)+\frac{\gamma}{2}\left(x-x_{0}\right)^{2}+o\left(\left(x-x_{0}\right)^{2}\right) .
$$

Hence, the point value is at most of order 2.

Proof. We may assume that $x_{0}=0$. We also can assume that $0 \notin \operatorname{supp} \hat{f}$ and that $\hat{f}$ is the derivative of a locally integrable function. Indeed, otherwise we express $\hat{f}=\hat{f}_{2}+\hat{f}_{1}$, where $\hat{f}_{2}$ is the derivative of a distribution with compact support, $0 \notin \operatorname{supp} \hat{f}_{1}$ and $\hat{f}_{1}$ is the first order derivative of a locally integrable function. Observe that $f_{2}$ is a $C^{\infty}$-function and $2 \pi f_{2}(0)=\left\langle\hat{f}_{2}(x), 1\right\rangle=$ 0 ; consequently, $f_{1}$ satisfies the hypothesis of the present theorem and $f$ satisfies the conclusions of the theorem if and only if $f_{1}$ does. 
The hypothesis implies that if $G$ is a primitive of $f$, then for each $a>0$,

$$
G(a x)-G(-x)=2 \pi \gamma+o(1) \text { as } x \rightarrow \infty \text {. }
$$

We choose $G$ such that $0 \notin \operatorname{supp} G$. Set $\sigma=G-\pi \gamma$, then $\sigma$ is asymptotically homogeneous of degree 0 , and

$$
G(x)=\pi \gamma \operatorname{sgn} x+\sigma(|x|)+o(1), \text { as }|x| \rightarrow \infty .
$$

By (4.4), $\sigma(x)=o(\log x)$. Therefore, $x^{-1} G(x) \in L^{2}(\mathbb{R})$ and $x^{-2} G(x) \in$ $L^{1}(\mathbb{R}) \cap L^{2}(\mathbb{R})$. Set,

$$
h(x)=\frac{1}{2 \pi} \int_{-\infty}^{\infty} e^{i x t} \frac{G(t)}{t^{2}} \mathrm{~d} t,
$$

then $h$ is continuous and $h(x)=o(1)$ as $|x| \rightarrow \infty$. We now relate $h$ to $f$, note that $h^{\prime \prime}=-\mathcal{F}^{-1}(G)$, so $i x h^{\prime \prime}(x)=f(x)$. In addition, we have that $h^{\prime}(x)=i \mathcal{F}^{-1}\left\{t^{-1} G(t) ; x\right\} \in L^{2}(\mathbb{R})$. Let $F_{2}$ be the following second order primitive of $f$,

$$
F_{2}(x)=i x h(x)-\frac{1}{\pi} \int_{-\infty}^{\infty} e^{i x t} \frac{G(t)}{t^{3}} \mathrm{~d} t
$$

Clearly, $F_{1}(x)=F_{2}^{\prime}(x)=i x h^{\prime}(x)-i h(x)$, which shows that every first order primitive of $f$ is locally integrable. We now show that $F_{2}$ is differentiable at 0 ,

$$
\frac{F_{2}(x)-F_{2}(0)}{x}=\frac{1}{2 \pi} \int_{-\infty}^{\infty} \frac{G(t)}{t^{2}}\left(\frac{i t x e^{i x t}-2 e^{i x t}+2}{t x}\right) \mathrm{d} t,
$$

we can apply Lebesgue Dominated Convergence Theorem in (5.2) to conclude that

$$
F_{2}^{\prime}(0)=-\frac{i}{2 \pi} \int_{-\infty}^{\infty} \frac{G(t)}{t^{2}} \mathrm{~d} t
$$

We now calculate the Peano second order differential of $F_{2}$ at 0 .

$$
\Delta^{2}(x)=\frac{F_{2}(x)-F_{2}(0)-x F_{2}^{\prime}(0)}{x^{2}}=\frac{x}{2 \pi} \int_{-\infty}^{\infty} G(t) K(x t) \mathrm{d} t,
$$

where $K(t)=t^{-3}\left(i t e^{i t}-2 e^{i t}+2+i t\right)$. Note that $(1+|\log (t)|) K(t)$ belongs to $L^{1}(\mathbb{R}) \cap L^{2}(\mathbb{R})$. Changing variables in (5.3) and applying Lemma 4.1 in combination with (5.1), we obtain that as $x \rightarrow 0$

$$
\begin{aligned}
\Delta^{2}(x) & =\frac{\operatorname{sgn} x}{2 \pi} \int_{-\infty}^{\infty} G\left(\frac{t}{x}\right) K(t) \mathrm{d} t \\
& =\frac{\gamma}{2} \int_{-\infty}^{\infty} \operatorname{sgn} t K(t) \mathrm{d} t+\frac{1}{2 \pi} \operatorname{sgn}(x) \sigma\left(|x|^{-1}\right) \int_{-\infty}^{\infty} K(t) \mathrm{d} t+o(1) \\
& =\frac{\gamma}{2}+o(1),
\end{aligned}
$$

since $\int_{0}^{\infty}(K(t)+K(-t) \mathrm{d} t)=0$ and $\int_{0}^{\infty}(K(t)-K(-t)) \mathrm{d} t=1$. This completes the proof.

We now use Theorem 5.1 to attack the general problem. 
Theorem 5.2. Let $f \in \mathcal{S}^{\prime}$. Suppose that

$$
\frac{1}{2 \pi} \text { e.v. }\left\langle\hat{f}(x), e^{i x_{0} x}\right\rangle=\gamma \quad(\mathrm{C}, k)
$$

then, $f\left(x_{0}\right)=\gamma$ in $\mathcal{S}^{\prime}, f$ is the derivative of order $k+1$ of a locally integrable function and the order of $f\left(x_{0}\right)=\gamma$ is less or equal to $k+2$.

Proof. As in the proof of the last theorem, we can assume that $x_{0}=0$, $0 \notin \operatorname{supp} \hat{f}$ and $\hat{f}$ is the derivative of order $k+1$ of a locally integrable function.

By our assumptions, we can choose $G$, a locally integrable function, such that $G^{k+1}=\hat{f}, 0 \notin \operatorname{supp} G$, and for each $a>0$,

$$
a^{-k} G(a x)+(-1)^{k+1} G(-x)=\frac{2 \pi \gamma}{k !} x^{k}+o\left(x^{k}\right) \quad \text { as } x \rightarrow \infty .
$$

Let $h$ be the following tempered distribution

$$
h(x)=-i x \mathcal{F}^{-1}\left\{t^{-k} G(t) ; x\right\}=\mathcal{F}^{-1}\left\{\left(t^{-k} G(t)\right)^{\prime} ; x\right\}
$$

note that $h$ satisfies the hypothesis of Theorem 5.1. Therefore, there is a locally integrable primitive $h_{1}$ of $h$ such that $h_{1}(\varepsilon x)=\gamma \varepsilon x / k !+o(\varepsilon)$ as $\varepsilon \rightarrow 0$ in $\mathcal{S}^{\prime}$. Set $h_{2}(x)=\int_{0}^{x} h_{1}(t) \mathrm{d} t$, then, by Theorem 5.1,

$$
h_{2}(x)=\frac{\gamma}{2 k !} x^{2}+o\left(x^{2}\right) \quad \text { as } x \rightarrow 0,
$$

since $h_{2}^{\prime}(0)$ is equal to the distributional point value of $h_{1}$ at 0 and $h_{1}(0)=0$ in $\mathcal{D}^{\prime}$. We now relate $h$ to $f$. We show that

$$
\begin{aligned}
F_{k+1}(x) & =\sum_{j=0}^{k} \frac{(-1)^{k-j} k !}{(k-j) !}\left(\begin{array}{c}
k+1 \\
j
\end{array}\right) I_{j}\left\{t^{k-j} h_{1}(t) ; x\right\} \\
& -\sum_{j=0}^{k} \frac{(-1)^{k-j} k !}{(k-j-1) !}\left(\begin{array}{c}
k+1 \\
j
\end{array}\right) I_{j+1}\left\{t^{k-j-1} h_{1}(t) ; x\right\}
\end{aligned}
$$


is a $(k+1)$-primitive of $f$. Differentiating $(5.5)(k+1)$ times, we obtain,

$$
\begin{aligned}
F_{k+1}^{(k+1)}(x) & =\sum_{j=0}^{k} \frac{(-1)^{k-j} k !}{(k-j) !}\left(\begin{array}{c}
k+1 \\
j
\end{array}\right) \frac{d^{k+1-j}}{d x^{k+1-j}}\left(x^{k-j} h_{1}(x)-(k-j) \int_{0}^{x} t^{k-j-1} h_{1}(t) \mathrm{d} t\right) \\
& =\sum_{j=0}^{k} \frac{(-1)^{k-j} k !}{(k-j) !}\left(\begin{array}{c}
k+1 \\
j
\end{array}\right) \frac{d^{k-j}}{d x^{k-j}}\left(x^{k-j} h(x)\right) \\
& =-i \sum_{j=0}^{k} \frac{(-1)^{k-j} k !}{(k-j) !}\left(\begin{array}{c}
k+1 \\
j
\end{array}\right) \frac{d^{k-j}}{d x^{k-j}}\left(x^{k+1-j} \mathcal{F}^{-1}\left\{G(t) / t^{k} ; x\right\}\right) \\
& =\sum_{j=0}^{k} \frac{(-i)^{k-j} k !}{(k-j) !}\left(\begin{array}{c}
k+1 \\
j
\end{array}\right) \frac{d^{k-j}}{d x^{k-j}}\left(\mathcal{F}^{-1}\left\{\left(G(t) / t^{k}\right)^{(k+1-j)} ; x\right\}\right) \\
& =\mathcal{F}^{-1}\left\{\sum_{j=0}^{k+1}\left(\begin{array}{c}
k+1 \\
j
\end{array}\right) \frac{d^{j}}{d t^{j}}\left(t^{k}\right)\left(G(t) / t^{k}\right)^{(k+1-j)} ; x\right\} \\
& =\mathcal{F}^{-1}\left\{G^{(k+1)}(t) ; x\right\}=\mathcal{F}^{-1}\{\hat{f}(t) ; x\}=f(x)
\end{aligned}
$$

Therefore, $F_{k+1}$ is a primitive of order $k+1$ of $f$. Since $h_{1}$ is locally integrable, so is $F_{k+1}$. We integrate (5.5) to obtain a continuous $(k+2)$-primitive of $f$, given by

$$
\begin{aligned}
& F_{k+2}(x)=\sum_{j=0}^{k} \frac{(-1)^{k-j} k !}{(k-j) !}\left(\begin{array}{c}
k+1 \\
j
\end{array}\right) I_{j}\left\{t^{k-j} h_{2}(t)-(k-j) \int_{0}^{t} s^{k-j-1} h_{2}(s) \mathrm{d} s ; x\right\} \\
& +\sum_{j=0}^{k} \frac{(-1)^{k-j} k !}{(k-j-1) !}\left(\begin{array}{c}
k+1 \\
j
\end{array}\right) I_{j+1}\left\{(k-j-1) \int_{0}^{t} s^{k-j-2} h_{2}(s) \mathrm{d} s-t^{k-j-1} h_{2}(t) ; x\right\} .
\end{aligned}
$$

By using (5.4), we can conclude that

$$
\begin{aligned}
F_{k+2}(x) & =\frac{\gamma}{2 k !} \sum_{j=0}^{k} \frac{(-1)^{k-j} k !}{(k-j) !}\left(\begin{array}{c}
k+1 \\
j
\end{array}\right) \frac{2 I_{j}\left\{t^{k+2-j} ; x\right\}}{(k+2-j)(k+1-j)}+o\left(x^{k+2}\right) \\
& =\frac{\gamma}{k !} x^{k+2} \sum_{j=0}^{k} \frac{(-1)^{k-j} k !}{(k-j) !}\left(\begin{array}{c}
k+1 \\
j
\end{array}\right) \frac{(k-j) !}{(k+2) !}+o\left(x^{k+2}\right) \\
& =\frac{\gamma}{k !(k+2)} x^{k+2} \sum_{j=0}^{k}(-1)^{k-j}\left(\begin{array}{c}
k \\
j
\end{array}\right) \frac{1}{(k+1-j)}+o\left(x^{k+2}\right) \\
& =\frac{\gamma}{k !(k+2)} x^{k+2}(-1)^{k} \int_{0}^{1}(t-1)^{k} d t+o\left(x^{k+2}\right) \\
& =\frac{\gamma}{(k+2) !} x^{k+2}+o\left(x^{k+2}\right) \text { as } x \rightarrow 0,
\end{aligned}
$$


this shows that $f(0)=\gamma$ in $\mathcal{S}^{\prime}$ and the order of the point value is at most $k+2$.

\section{Symmetric point values}

This section is devoted to the study of symmetric point values of distributions. They are studied by means of the symmetric part of a distribution about at given point $x=x_{0}$, that is, the distribution

$$
\chi_{x_{0}}^{f}(x):=\frac{f\left(x+x_{0}\right)+f\left(x_{0}-x\right)}{2}
$$

Notice that $\chi_{x_{0}}^{f}$ is an even distribution.

Definition 6.1. Let $f \in \mathcal{D}^{\prime}$ and $x_{0} \in \mathbb{R}$. We say that $f$ has a (distributional) symmetric point value $\gamma$ at $x=x_{0}$ if its symmetric part about $x_{0}$ has a point value at $x=0$, that is, $\chi_{x_{0}}^{f}(0)=\gamma$ in $\mathcal{D}^{\prime}$. In this case we write $f_{\text {sym }}\left(x_{0}\right)=\gamma$ in $\mathcal{D}^{\prime}$.

Of course, the existence of the symmetric value at $x_{0}$ is equivalent to have

$$
\lim _{\varepsilon \rightarrow 0^{+}} \frac{1}{2 \varepsilon}\left\langle f(x), \phi\left(\frac{x-x_{0}}{\varepsilon}\right)+\phi\left(\frac{x_{0}-x}{\varepsilon}\right)\right\rangle=\gamma \int_{-\infty}^{\infty} \phi(x) \mathrm{d} x,
$$

for each $\phi \in \mathcal{D}^{\prime}$. We may use Eojasiewicz characterization of distributional point values (2.4) to characterize symmetric point values.

Theorem 6.2. Let $f \in \mathcal{D}^{\prime}$ and $x_{0} \in \mathbb{R}$. We have that $f_{\text {sym }}\left(x_{0}\right)=\gamma$ in $\mathcal{D}^{\prime}$ if and only if there exists $n \in \mathbb{N}$ and an n-primitive $F$ of $f$ such that $F\left(x_{0}+x\right)+(-1)^{n} F\left(x_{0}-x\right)$ is locally integrable in a neighborhood of the origin and

$$
F\left(x_{0}+h\right)+(-1)^{n} F\left(x_{0}-h\right)=2 \gamma \frac{h^{n}}{n !}+o\left(h^{n}\right), \quad h \rightarrow 0 .
$$

Most of the results for symmetric point values can be obtained from those of distributional point values. Let us discuss an example.

Example 6.3. Let $f \in \mathcal{D}^{\prime}$ have a distributional point value $\gamma$ at $x_{0}$. Let $U$ be a harmonic representation [1] of $f$ on $\Im m z>0$, that is, $U(z)$ is harmonic for $\Im m z>0$ and $\lim _{y \rightarrow 0^{+}} U(x+i y)=f(x)$ in the weak topology of $\mathcal{D}^{\prime}$. It is shown in [8] by studying the Poisson kernel and in [34] by Fourier transform methods that

$$
\lim _{z \rightarrow x_{0}} U(z)=\gamma, \quad \text { non-tangentially from the upper half-plane. }
$$

We can use this result applied to the symmetric distribution to obtain a radial version of this result in the case of symmetric point values. Indeed, suppose now that $f_{\text {sym }}\left(x_{0}\right)=\gamma$ in $\mathcal{D}^{\prime}$. If $U$ is a harmonic representation of $f$. Then $U_{1}(z)=\left(U\left(x_{0}+z\right)+U\left(x_{0}-\bar{z}\right)\right) / 2$ is a harmonic representation of 
$\chi_{x_{0}}^{f}$, hence $U_{1}(z)=\gamma+o(1)$ as $z$ approaches 0 from the upper half-plane in a non-tangential manner. Therefore,

$$
\lim _{y \rightarrow 0^{+}} U\left(x_{0}+i y\right)=\lim _{y \rightarrow 0^{+}} U_{1}(i y)=\gamma .
$$

In particular, if $f$ is a $2 \pi$-periodic distribution with sines and cosines series $f(x)=a_{0} / 2+\sum_{n=1}^{\infty} a_{n} \cos n x+b_{n} \sin n x$, then

$$
\frac{a_{0}}{2}+\sum_{n=1}^{\infty}\left(a_{n} \cos n x_{0}+b_{n} \sin n x_{0}\right)=\gamma \quad(\mathrm{A})
$$

where (A) stands for Abel summability [15]. This recovers the main result from $[43]$.

We say that $f_{\text {sym }}\left(x_{0}\right)=\gamma$ in $\mathcal{S}^{\prime}$ if $\chi_{x_{0}}^{f} \in \mathcal{S}^{\prime}$ and (6.2) holds for each $\phi \in \mathcal{S}$. It is then obvious that if $\chi_{x_{0}}^{f} \in \mathcal{S}^{\prime}$, we have that $f_{\text {sym }}\left(x_{0}\right)=\gamma$ in $\mathcal{D}^{\prime}$ if and only if $f_{\text {sym }}\left(x_{0}\right)=\gamma$ in $\mathcal{S}^{\prime}$.

In the same manner as for distributional point values, we define the order of symmetric point values.

Definition 6.4. Let $f \in \mathcal{D}^{\prime}$. Suppose that $\chi_{x_{0}}^{f} \in \mathcal{S}^{\prime}$. We say that $f$ has a (distributional) symmetric point value $\gamma$ at $x_{0}$ in $\mathcal{S}^{\prime}$ of order $n$, and write $f_{\text {sym }}\left(x_{0}\right)=\gamma$ in $\mathcal{S}^{\prime}$ with order $n$, if $n$ is the minimum integer such that there exists a distribution $F$ such that $F^{(n)}=f, F\left(x_{0}+x\right)+(-1)^{n} F\left(x_{0}-x\right)$ is a locally bounded measurable function of polynomial growth at infinity and $F$ satisfies (6.3).

We can also describe distributional symmetric point values in terms of $d e$ la Vallée Poussin derivatives [32], [46, Chapter XI]. Given a distribution $f$ define its jump distribution at $x=x_{0}$ by

$$
\psi_{x_{0}}^{f}(x)=\frac{f\left(x_{0}+x\right)-f\left(x_{0}-x\right)}{2} .
$$

Then, in the case that $n$ is even in Theorem 6.2 , we obtain that $\chi_{x_{0}}^{F}(h)=$ $\gamma h^{n} / n !+o\left(h^{n}\right)$; but on the other hand when $n$ is odd $\psi_{x_{0}}^{F}(h)=\gamma h^{n} / n !+$ $o\left(h^{n}\right)$. Let now $F_{1}$ be an arbitrary $n$-primitive of $f$, then we obtain that $F_{1}$ is de la Vallée Poussin $n$-differentiable at $x=x_{0}$, that is, either

$$
\chi_{x_{0}}^{F_{1}}(h)=a_{0}+a_{2} h^{2}+\cdots+\gamma h^{n} / n !+o\left(h^{n}\right), \quad \text { as } h \rightarrow 0,
$$

for some constants $a_{0}, a_{2} \ldots$, when $n$ is even, or

$$
\psi_{x_{0}}^{F_{1}}(h)=b_{1} h+b_{3} h^{3}+\cdots+\gamma h^{n} / n !+o\left(h^{n}\right), \quad \text { as } h \rightarrow 0,
$$

for some constants $b_{1}, b_{3} \ldots$, when $n$ is odd.

Our main goal in this section is to formulate and solve the problem of (C) symmetric summability for tempered distributions. We will do this with the aid of symmetric point values. Let us first discuss a known case, namely, Fourier series. 
Example 6.5. Suppose that $f$ is a $2 \pi$-periodic distribution with sines and cosines Fourier series $f(x)=a_{0} / 2+\sum_{n=1}^{\infty}\left(a_{n} \cos n x+b_{n} \sin n x\right)$. Then the solution to the $(\mathrm{C})$ problem (see the Introduction) can be formulated in terms of symmetric point values. Indeed, [9, Theorem 6.14.4], [16],

$$
f(x)=\frac{a_{0}}{2}+\sum_{n=1}^{\infty}\left(a_{n} \cos n x_{0}+b_{n} \sin n x_{0}\right)=\gamma
$$

if and only if $f_{\text {sym }}\left(x_{0}\right)=\gamma$ in $\mathcal{D}^{\prime}$. In $[14,45]$, using the language of de la Vallée Poussin derivatives the order of summability is estimated upon knowledge of the order of the point value; the opposite problem was investigated in [46, Chapter XI, Theorem 2.1] (the original source is [45]).

The natural extension of the Hardy-Littlewood (C) summability problem is then to characterize symmetric point values of tempered distributions by summability of the Fourier transform. In order to characterize symmetric point values in terms of the Fourier transform, we need to choose the correct notion of summability. It turns out the right notion is that of principal value distributional evaluations in the $(\mathrm{C})$ sense, which we now proceed to define.

Definition 6.6. Let $g \in \mathcal{D}^{\prime}, \phi \in C^{\infty}(\mathbb{R})$ and $\beta \geq 0$. We say that the evaluation $\langle g(x), \phi(x)\rangle$ exists in the p.v. Cesàro sense (at order $\beta$ ), and write

$$
\text { p.v. }\langle g(x), \phi(x)\rangle=\gamma \quad(\mathrm{C}, \beta),
$$

if for some primitive $G$ of $g \phi$ we have

$$
\lim _{x \rightarrow \infty}(G(x)-G(-x))=\gamma \quad(\mathrm{C}, \beta) .
$$

If (6.5) exits, we also say that the principal value of the evaluation exists in the $(\mathrm{C}, \beta)$ sense.

Suppose that $g$ is locally integrable, then we write (6.5) as

$$
\text { p.v. } \int_{-\infty}^{\infty} g(x) \phi(x) \mathrm{d} x=\gamma \quad(\mathrm{C}, \beta) .
$$

and we say that the principal value of the integral exists. Notice that (6.6) exactly means

$$
\lim _{x \rightarrow \infty} \int_{-x}^{x} g(t) \phi(t)\left(1-\frac{|t|}{x}\right)^{\beta} \mathrm{d} t=\gamma .
$$

For numerical series, let $\left\{c_{n}\right\}_{n=-\infty}^{\infty}$ be a sequence of complex numbers, we say that the principal value of the series exits in the $(\mathrm{C}, \beta)$ sense and write

$$
\text { p.v. } \sum_{n=-\infty}^{\infty} c_{n} \phi(n)=\gamma \quad(\mathrm{C}, \beta),
$$


if

$$
c_{0}+\sum_{n=1}^{\infty}\left(c_{n}+c_{-n}\right) \phi(n)=\gamma \quad(\mathrm{C}, \beta),
$$

where in the last equation $(\mathrm{C}, \beta)$ stands for Cesàro summability but in the sense of numerical series [15]. Because of the equivalence between Cesàro means and Riesz typical means $[15,18,28]$, it means that

$$
\lim _{x \rightarrow \infty} \sum_{-x<n<x} c_{n} \phi(n)\left(1-\frac{|n|}{x}\right)^{\beta}=\gamma ;
$$

thus, (6.7) is equivalent to have (6.5) with $g(x)=\sum_{n=-\infty}^{\infty} c_{n} \delta(x-n)$.

We easily obtain a version of Theorem 3.6 for symmetric point values.

Theorem 6.7. Let $f \in \mathcal{D}^{\prime}$. Suppose that there exists an $m \in \mathbb{N}$, such that every $m$-primitive of $\chi_{x_{0}}^{f}$ is a locally bounded measurable function for large arguments and satisfies an estimate $O\left(|x|^{m-1}\right)$, as $x \rightarrow \infty$. If $f_{\mathrm{sym}}\left(x_{0}\right)=\gamma$ in $\mathcal{S}^{\prime}$ with order $n$, then

$$
\frac{1}{2 \pi} \mathrm{p} \cdot \mathrm{v} \cdot\left\langle\hat{\chi}_{x_{0}}^{f}(x), 1\right\rangle=\gamma \quad(\mathrm{C}, \beta),
$$

for any $\beta>k=\max \{m, n\}$. When $f \in \mathcal{S}^{\prime}$, we obtain

$$
\frac{1}{2 \pi} \mathrm{p} \cdot \mathrm{v} \cdot\left\langle\hat{f}(x), e^{i x_{0} x}\right\rangle=\gamma \quad(\mathrm{C}, \beta)
$$

for any $\beta>k=\max \{m, n\}$.

Proof. Our hypotheses imply that $\chi_{x_{0}}^{f} \in \mathcal{S}^{\prime}$, thus we can apply Theorem 3.6 to $\chi_{x_{0}}^{f}$. Since, $\chi_{x_{0}}^{f}(0)=\gamma$ in $\mathcal{S}^{\prime}$ with order $n$, then

$$
\text { e.v. }\left\langle\hat{\chi}_{x_{0}}^{f}(x), 1\right\rangle=2 \pi \gamma \quad(\mathrm{C}, \beta)
$$

for any $\beta>k=\max \{m, n\}$, in particular the last relation holds in the p.v. sense. If we assume that $f \in \mathcal{S}^{\prime}$, then

$$
\hat{\chi}_{x_{0}}^{f}(x)=\frac{1}{2}\left(e^{i x_{0} x} \hat{f}(x)+e^{-i x_{0} x} \hat{f}(-x)\right),
$$

so, if $F$ is first order primitive of $e^{i x_{0} x} \hat{f}(x)$, then $G(x)=(F(x)-F(-x)) / 2$ is a first order primitive of $\hat{\chi}_{x_{0}}^{f}(x)$, and hence

$$
\lim _{x \rightarrow \infty}(G(x)-G(-x))=\lim _{x \rightarrow \infty}(F(x)-F(-x))=2 \pi \gamma \quad(\mathrm{C}, \beta) \text {. }
$$

When $f$ has compact support we obtain the following result.

Corollary 6.8. Let $f$ be a distribution with compact support. Suppose that $f_{\text {sym }}\left(x_{0}\right)=\gamma$ in $\mathcal{S}^{\prime}$ with order $k$. Then for any $\beta>k$,

$$
\frac{1}{2 \pi} \text { p.v. } \int_{-\infty}^{\infty} \hat{f}(t) e^{i x_{0} t} \mathrm{~d} t=\gamma \quad(\mathrm{C}, \beta),
$$


or which is the same

$$
\lim _{x \rightarrow \infty} \frac{1}{2 \pi} \int_{-x}^{x}\left(1-\frac{|t|}{x}\right)^{\beta} \hat{f}(t) e^{i x_{0} t} \mathrm{~d} t=\gamma .
$$

For Fourier series, we obtain the result of A. Zygmund [45] mentioned in Example 6.5. Obviously, our language differers from that of Zygmund's original statement.

Corollary 6.9. Let $f$ be a $2 \pi$-periodic distribution, with sines and cosines Fourier series

$$
f(x)=\frac{a_{0}}{2}+\sum_{n=1}^{\infty}\left(a_{n} \cos n x+b_{n} \sin n x\right) .
$$

Suppose that $f_{\mathrm{sym}}\left(x_{0}\right)=\gamma$ in $\mathcal{S}^{\prime}$ with order $k \geq 0$. Then for any $\beta>k$,

$$
\frac{a_{0}}{2}+\sum_{n=1}^{\infty}\left(a_{n} \cos n x_{0}+b_{n} \sin n x_{0}\right)=\gamma \quad(\mathrm{C}, \beta)
$$

or equivalently

$$
\lim _{x \rightarrow \infty} \frac{a_{0}}{2}+\sum_{0<n<x}\left(1-\frac{|n|}{x}\right)^{\beta}\left(a_{n} \cos n x_{0}+b_{n} \sin n x_{0}\right)=\gamma .
$$

Proof. If $k \geq 1$, we can assume that $a_{0}=0$ and proceed to apply Theorem 6.7. For $k=0$, then $f$ is a bounded $2 \pi$-periodic function which is continuous at $x_{0}$, and hence the conclusion follows from the classical result $[19,46]$.

As in the proof of Theorem 6.7, one can apply the result for distributional point values, Theorem 3.14 , to the distribution $\chi_{x_{0}}^{f}$ to easily obtain the next bound for the order of summability in the case of the principal value of Fourier inversion formula for general tempered distributions.

Theorem 6.10. Let $f \in \mathcal{D}^{\prime}$. Suppose that

$$
\chi_{x_{0}}^{f}(x)=O\left(|x|^{\alpha}\right) \quad(\mathrm{C}, m), \text { as }|x| \rightarrow \infty .
$$

If $f_{\text {sym }}\left(x_{0}\right)=\gamma$ in $\mathcal{S}^{\prime}$ with order $n$, then

$$
\frac{1}{2 \pi} \mathrm{p} \cdot \mathrm{v} \cdot\left\langle\hat{\chi}_{x_{0}}^{f}(x), 1\right\rangle=\gamma \quad(\mathrm{C}, k+1),
$$

where $k=\max \left\{m, n,\left[n+\alpha+\frac{1}{2}\right],\left[m+\alpha+\frac{1}{2}\right]\right\}$. If we assume $f \in \mathcal{S}^{\prime}$, then we obtain

$$
\frac{1}{2 \pi} \text { p.v. }\left\langle\hat{f}(x), e^{i x_{0} x}\right\rangle=\gamma \quad(\mathrm{C}, k+1) .
$$

Finally, we estimate the order of the symmetric point value in terms of the order of summability of the principal value Fourier inversion formula. We need the following lemma. 
Lemma 6.11. Let $g \in \mathcal{D}^{\prime}$ be an even distribution, that is, $g(-x)=g(x)$, then

$$
\text { e.v. }\langle g(x), 1\rangle=\gamma \quad(\mathrm{C}, \beta)
$$

if and only if

$$
\text { p.v. }\langle g(x), 1\rangle=\gamma \quad(\mathrm{C}, \beta) .
$$

In fact the above relations are equivalent to

$$
\lim _{x \rightarrow \infty} G(x)=\frac{\gamma}{2} \quad(\mathrm{C}, \beta),
$$

where $G$ is the unique odd first order primitive of $g$.

Proof. That (6.9) and (6.10) are equivalent is clear. Relation (6.8) obviously implies (6.9). We now show that (6.10) implies (6.8). Let $G$ be the odd first order primitive of $g$, so we have that $G(x)=\gamma / 2+o(1)(\mathrm{C}, \beta)$ as $x \rightarrow \infty$, hence we also have that $G(a x)=\gamma / 2+o(1)(\mathrm{C}, \beta)$ as $x \rightarrow \infty$, and thus for each $a>0$

$$
\lim _{x \rightarrow \infty}(G(a x)-G(-x))=2 \lim _{x \rightarrow \infty}(G(a x)+G(x))=\gamma \quad(\mathrm{C}, \beta) .
$$

Therefore, on combining Lemma 6.11 and Theorem 5.2, we immediately obtain the following result. Notice that, as a corollary, we obtain the classical result [46, Chapter XI, Theorem 2.1] for Fourier series.

Theorem 6.12. Let $f \in \mathcal{S}^{\prime}$. Suppose that

$$
\frac{1}{2 \pi} \mathrm{p} \cdot \mathrm{v} \cdot\left\langle\hat{f}(x), e^{i x_{0} x}\right\rangle=\gamma \quad(\mathrm{C}, k),
$$

then, $f_{\mathrm{sym}}\left(x_{0}\right)=\gamma$ in $\mathcal{S}^{\prime}, \chi_{x_{0}}^{f}$ is the derivative of order $k+1$ of a locally integrable function and the order of $f_{\mathrm{sym}}\left(x_{0}\right)$ is less or equal to $k+2$.

The solution of the $(\mathrm{C})$ symmetric problem for "trigonometric integrals" of distributions is summarized in the last theorem of this section, which extends Hardy and Littlewood characterization [16] of (symmetric) (C) summability at a point from Fourier series to general tempered distributions.

Theorem 6.13. Let $f \in \mathcal{D}^{\prime}$. Suppose that $\chi_{x_{0}}^{f} \in \mathcal{S}^{\prime}$. Then

$$
\frac{1}{2 \pi} \mathrm{p} \cdot \mathrm{v} \cdot\left\langle\hat{\chi}_{x_{0}}^{f}, 1\right\rangle=\gamma
$$

if and only if $f_{\mathrm{sym}}\left(x_{0}\right)=\gamma$ in $\mathcal{D}^{\prime}$. If in addition $f \in \mathcal{S}^{\prime}$, then (6.11) is the same as

$$
\frac{1}{2 \pi} \text { p.v. }\left\langle\hat{f}(x), e^{i x_{0} x}\right\rangle=\gamma \quad(\mathrm{C})
$$




\section{THE ORDER OF JUMPS AND SYMMETRIC JUMPS}

In this last section we study the order of summability in several characterizations the author have obtained for the jump behavior and symmetric jump behavior of distributions $[35,36,37,10]$. Let us define these two concepts.

Definition 7.1. A distribution $f \in \mathcal{D}^{\prime}$ is said to have a distributional jump behavior (or jump behavior) at $x=x_{0} \in \mathbb{R}$ if it satisfies the following distributional asymptotic relation

$$
f\left(x_{0}+\varepsilon x\right)=\gamma_{-} H(-x)+\gamma_{+} H(x)+o(1),
$$

as $\varepsilon \rightarrow 0^{+}$in $\mathcal{D}^{\prime}$, where $H$ is the Heaviside function, i.e., the characteristic function of $(0, \infty)$, and $\gamma_{ \pm}$are constants. The jump (or saltus) of $f$ at $x=x_{0}$ is defined then as the number $[f]_{x=x_{0}}=\gamma_{+}-\gamma_{-}$.

The meaning of (7.1) is in the weak topology of $\mathcal{D}^{\prime}$, in the sense that for each $\phi \in \mathcal{D}$,

$$
\lim _{\varepsilon \rightarrow 0^{+}}\left\langle f\left(x_{0}+\varepsilon x\right), \phi(x)\right\rangle=\gamma_{-} \int_{-\infty}^{0} \phi(x) \mathrm{d} x+\gamma_{+} \int_{0}^{\infty} \phi(x) \mathrm{d} x .
$$

If $f \in \mathcal{S}^{\prime}$, then it is shown in [39] that (7.2) remains true for all $\phi \in \mathcal{S}^{\prime}$. Furthermore, from the results of [39], a structural characterization of the jump behavior of distributions can be given explicitly, that is, a distribution $f \in \mathcal{D}^{\prime}$ has the jump behavior (7.1) if and only if there exist $n \in \mathbb{N}$ and a function $F$, locally integrable on a neighborhood of $x_{0}$, such that $F^{(n)}=f$ near $x_{0}$ and

$$
\lim _{x \rightarrow x_{0}^{ \pm}} \frac{n ! F(x)}{\left(x-x_{0}\right)^{n}}=\gamma_{ \pm} .
$$

So we can define the order of the jump behavior in $\mathcal{S}^{\prime}$ of a tempered distribution.

Definition 7.2. Let $f \in \mathcal{S}^{\prime}$. Suppose that $f$ has jump behavior at $x_{0}$. The order of the jump behavior in $\mathcal{S}^{\prime}$ is defined as the minimum integer $n$ such that there exists a locally bounded measurable function $F$ of polynomial growth at infinity satisfying $F^{(n)}=f$ and (7.3).

Recall the definition of the jump distribution of $f$ at $x=x_{0}$, it is given by $(6.4)$.

Definition 7.3. A distribution $f \in \mathcal{D}^{\prime}$ is said to have a distributional symmetric jump behavior (or symmetric jump behavior) at $x=x_{0} \in \mathbb{R}$ if the jump distribution $\psi_{x_{0}}^{f}$ has jump behavior at 0 . In such a case, we define the symmetric jump (or saltus) of $f$ at $x=x_{0}$ as the number $[f]_{x=x_{0}}=\left[\psi_{x_{0}}^{f}\right]_{x=0}$ . We say that $f$ has a jump behavior in $\mathcal{S}^{\prime}$ with order $n$ if $\psi_{x_{0}}^{f} \in \mathcal{S}^{\prime}$ and it has a jump behavior at $x=0$ of order $n$. 
Since the jump distribution is odd, it is easy to see that the jump behavior of the jump distribution in Definition 7.3 must be of the form

$$
\psi_{x_{0}}(\varepsilon x)=\frac{1}{2}[f]_{x=x_{0}} \operatorname{sgn} x+o(1) \quad \text { as } \varepsilon \rightarrow 0^{+} \text {in } \mathcal{D}^{\prime},
$$

where $\operatorname{sgn} x$ is the signum function, i.e., $H(x)-H(-x)$. Notice that a distribution $f$ has jump behavior (7.1) at $x=x_{0}$ if and only if it has symmetric point value and symmetric jump behavior at $x=x_{0}$ and $f_{\mathrm{sym}}\left(x_{0}\right)=$ $\left(\gamma_{-}+\gamma_{+}\right) / 2$ and $[f]_{x=x_{0}}=\gamma_{+}-\gamma_{-}$.

We now add information about the order to the characterization of the jump behavior given in $[35,36]$.

Theorem 7.4. Let $f \in \mathcal{S}^{\prime}$ have the distributional jump behavior (7.1) at $x=x_{0}$ of order $n$. Suppose that there exists an $m \in \mathbb{N}$ such that every $m$-primitive of $f$ is a locally integrable function for large arguments and satisfies an estimate $O\left(|x|^{m-1}\right)$, as $x \rightarrow \infty$. Let $F$ be a fist order primitive of $e^{i x_{0} x} \hat{f}$, then if $\beta>k=\max \{m, n\}$,

$$
\frac{1}{2 \pi} \lim _{x \rightarrow \infty}(F(a x)-F(-x))=f_{\mathrm{sym}}\left(x_{0}\right)+\frac{[f]_{x=x_{0}}}{2 \pi i} \log a \quad(\mathrm{C}, \beta),
$$

uniformly for a in compact subsets of $(0, \infty)$.

Proof. Define the distribution

$$
v=-\theta_{[-1,0]}+\theta_{[0,1]} .
$$

Then the distribution

$$
h(x)=f(x)-\frac{1}{2}[f]_{x=0} v\left(x-x_{0}\right)
$$

satisfies the hypothesis of Theorem 3.6 and $h\left(x_{0}\right)=f_{\text {sym }}\left(x_{0}\right)$ in $\mathcal{S}^{\prime}$ with order $n$. Therefore,

$$
\text { e.v. }\left\langle\hat{h}(x), e^{i x_{0} x}\right\rangle=2 \pi f_{\mathrm{sym}}\left(x_{0}\right) \quad(\mathrm{C}, \beta),
$$

whenever $\beta>k=\max \{m, n\}$. Observe that

$$
e^{i x_{0} x} \mathcal{F}\left\{v\left(t-x_{0}\right) ; x\right\}=\hat{v}(x)=\frac{2-2 \cos x}{i x} .
$$

Let $\mathrm{G}$ be a first order primitive of $e^{i x_{0} x} \hat{h}(x)$, hence

$$
F(x)=G(x)+\frac{[f]_{x=x_{0}}}{i} \int_{0}^{x} \frac{1-\cos t}{t} \mathrm{~d} t
$$


satisfies $F^{\prime}(x)=e^{i x_{0} x} \hat{f}(x)$. Then, we obtain as $x \rightarrow \infty$

$$
\begin{aligned}
F(a x)-F(-x) & =G(a x)-G(-x)+\frac{[f]_{x=x_{0}}}{i} \int_{-x}^{a x} \frac{1-\cos t}{t} \mathrm{~d} t \\
& =2 \pi f_{\mathrm{sym}}\left(x_{0}\right)+\frac{[f]_{x=x_{0}}}{i} \int_{x}^{a x} \frac{1-\cos t}{t} \mathrm{~d} t+o(1) \\
& =2 \pi f_{\mathrm{sym}}\left(x_{0}\right)+\frac{[f]_{x=x_{0}}}{i} \log a+o(1) \quad(\mathrm{C}, \beta) .
\end{aligned}
$$

We obtain immediately form Theorem 7.4 the corresponding results for compactly supported distributions and Fourier series. Here we only state the result for Fourier series and leave the corresponding statement for compactly supported distributions to the reader.

Corollary 7.5. Let $f$ be a $2 \pi$-periodic distribution, with Fourier series $\sum_{n=-\infty}^{\infty} c_{n} e^{i x n}$. Suppose that $f$ has the distributional jump behavior (7.1) at $x=x_{0}$ in $\mathcal{S}^{\prime}$ with order $k \geq 1$. Then for each $a>0$ and $\beta>k$,

$$
\lim _{x \rightarrow \infty} \sum_{-x \leq n \leq a x} c_{n} e^{i x_{0} n}=f_{\mathrm{sym}}\left(x_{0}\right)+\frac{[f]_{x=x_{0}}}{2 \pi i} \log a \quad(\mathrm{C}, \beta)
$$

or equivalently

$$
\lim _{x \rightarrow \infty} \sum_{-x \leq n \leq a x} \phi_{a}^{\beta}\left(\frac{n}{x}\right) c_{n} e^{i x_{0} n}=f_{\mathrm{sym}}\left(x_{0}\right)+\frac{[f]_{x=x_{0}}}{2 \pi i} \log a .
$$

Moreover, relation (7.4) holds uniformly for a in compact subsets of $(0, \infty)$.

Using the same procedure as in the proof of Theorem 7.4, we obtain from Theorem 3.14 and Theorem 5.2.

Theorem 7.6. Let $f \in \mathcal{S}^{\prime}$ have the distributional jump behavior (7.1) at $x=x_{0}$ of order $n$. Suppose that

$$
f(x)=O\left(|x|^{\alpha}\right) \quad(\mathrm{C}, m), \text { as }|x| \rightarrow \infty .
$$

Let $F$ be a first order primitive of $e^{i x_{0} x} \hat{f}(x)$. Then we have, uniformly for a in compact subset of $(0, \infty)$,

$$
\frac{1}{2 \pi} \lim _{x \rightarrow \infty}(F(a x)-F(-x))=f_{\mathrm{sym}}\left(x_{0}\right)+\frac{[f]_{x=x_{0}}}{2 \pi i} \log a \quad(\mathrm{C}, k+1),
$$

where $k=\max \left\{m, n,\left[n+\alpha+\frac{1}{2}\right],\left[m+\alpha+\frac{1}{2}\right]\right\}$.

Theorem 7.7. Let $f \in \mathcal{S}^{\prime}$. Let $F$ be a first order primitive of $f$. Suppose that for some constants $d_{1}$ and $d_{2}$

$$
\frac{1}{2 \pi} \lim _{x \rightarrow \infty}(F(a x)-F(-x))=d_{1}+d_{2} \log a \quad(\mathrm{C}, k),
$$

for a in a subset of positive measure of the interval $(0, \infty)$. Then, $f$ has the distributional jump behavior (7.1) at $x_{0}$ with constants $\gamma_{ \pm}=d_{1} \pm i \pi d_{2}, f$ is 
the derivative of order $k+1$ of a locally integrable function and the order of the jump behavior is less or equal to $k+2$.

It is possible to formulate analogous results for the symmetric jump behavior in terms of the jump distribution; however, we choose only to do it for the case of Fourier series.

Theorem 7.8. Let $f$ be a $2 \pi$-distribution with Fourier series

$$
f(x)=\frac{a_{0}}{2}+\sum_{n=1}^{\infty}\left(a_{n} \cos n x+b_{n} \sin n x\right) .
$$

Suppose that $f$ has a symmetric jump behavior at $x=x_{0}$ of order $k \geq 1$. Then for any $\beta>k$

$$
\lim _{x \rightarrow \infty} \sum_{x<n \leq a x}\left(a_{n} \sin n x_{0}-b_{n} \cos n x_{0}\right)=-\frac{[f]_{x=x_{0}}}{\pi} \log a \quad(\mathrm{C}, \beta),
$$

uniformly for a in compact subsets of $[1, \infty)$.

Proof. The jump distribution has Fourier transform

$$
\psi_{x_{0}}^{f}(x)=-\sum_{n=1}^{\infty}\left(a_{n} \sin n x_{0}-b_{n} \cos n x_{0}\right) \sin n x,
$$

it has Fourier transform

$$
\hat{\psi}_{x_{0}}^{f}(x)=\pi i \sum_{n=1}^{\infty}\left(a_{n} \sin n x_{0}-b_{n} \cos n x_{0}\right)(\delta(x-n)-\delta(x+n)) .
$$

Therefore,

$$
\Psi(x)=\pi i \sum_{1 \leq n<|x|}\left(a_{n} \sin n x_{0}-b_{n} \cos n x_{0}\right)
$$

is a first order primitive of the $\hat{\psi}_{x_{0}}^{f}$. Since it has a jump behavior at $x=0$ with jump $[f]_{x=x_{0}}$, Theorem 7.4 implies the result.

Reasoning as in Theorem 7.8, we can prove using Theorem 7.7 the following result.

Theorem 7.9. Let $f$ be a $2 \pi$-distribution with Fourier series

$$
f(x)=\frac{a_{0}}{2}+\sum_{n=0}^{\infty}\left(a_{n} \cos n x+b_{n} \sin n x\right) .
$$

Suppose that

$$
\lim _{x \rightarrow \infty} \sum_{x<n \leq a x}\left(a_{n} \sin n x_{0}-b_{n} \cos n x_{0}\right)=d \log a \quad(\mathrm{C}, k),
$$

for $a$ in a subset of positive measure of the interval $[1, \infty)$. Then, $f$ has the distributional symmetric jump behavior at $x_{0}$ with jump $[f]_{x=x_{0}}=-\pi d, \psi_{x_{0}}^{f}$ is the derivative of order $k+1$ of a locally integrable function and the order of the jump behavior is less or equal to $k+2$. 
We may use Theorems 7.8, Theorem 7.9 and Corollary 6.9 to characterize the distributional jump behavior of a $2 \pi$-periodic distribution from its sines and cosines Fourier series and its conjugate series.

Theorem 7.10. Let $f$ be a $2 \pi$-distribution with Fourier series

$$
f(x)=\frac{a_{0}}{2}+\sum_{n=0}^{\infty}\left(a_{n} \cos n x+b_{n} \sin n x\right) .
$$

Then $f$ has distributional jump behavior at $x=x_{0}$ if and only if there exists $\beta \geq 0$ such that for some constants $d_{1}$ and $d_{2}$

$$
\frac{a_{0}}{2}+\sum_{n=0}^{\infty}\left(a_{n} \cos n x_{0}+b_{n} \sin n x_{0}\right)=d_{1} \quad(\mathrm{C}, \beta),
$$

and

$$
\lim _{x \rightarrow \infty} \sum_{x<n \leq a x}\left(a_{n} \sin n x_{0}-b_{n} \cos n x_{0}\right)=d_{2} \log a \quad(\mathrm{C}, \beta),
$$

for $a$ in a subset of positive measure of the interval $[1, \infty)$. In such case $f_{\text {sym }}\left(x_{0}\right)=d_{1}$ and $[f]_{x=x_{0}}=-\pi d_{2}$.

The last results we want to comment are in relation with the classical formula of F. Lukács for the jump of a function [22, 24, 25]. Indeed, exactly the same arguments given in [37] now in combination with the information about the order from Theorem 7.4, Corollary 7.5 and Theorem 7.6 yield the following series of results.

Theorem 7.11. Let $f \in \mathcal{S}^{\prime}$ have the distributional jump behavior at $x=x_{0}$ of order $n$. Suppose that there exists a $m \in \mathbb{N}$, such that every $m$-primitive of $f$ is a locally integrable function for large arguments and satisfies an estimate $O\left(|x|^{m-1}\right)$, as $x \rightarrow \infty$. Then for any decomposition $\hat{f}=\hat{f}_{-}+\hat{f}_{+}$, where $\operatorname{supp} \hat{f}_{-} \subseteq(-\infty, 0]$ and $\operatorname{supp} \hat{f}_{+} \subseteq[0, \infty)$, and for any $\beta>\max \{n, m\}$, we have that the following convolutions are locally bounded functions and

$$
\left(e^{ \pm i x_{0} t} \hat{f}_{ \pm}( \pm t) * t_{+}^{\beta}\right)(x) \sim \pm[f]_{x=x_{0}} \frac{|x|^{\beta}}{i} \log x, \quad \text { as } x \rightarrow \infty,
$$

in the ordinary sense.

Theorem 7.12. Let $f$ be a $2 \pi$-periodic distribution, with Fourier series $\sum_{n=-\infty}^{\infty} c_{n} e^{i x n}$. Suppose it has distributional jump behavior at $x=x_{0}$ of order $k \geq 1$. Then for any $\beta>k$

$$
\lim _{x \rightarrow \infty} \frac{1}{\log x} \sum_{0 \leq n \leq x} c_{ \pm n} e^{ \pm i n x_{0}}\left(1-\frac{n}{x}\right)^{\beta}= \pm \frac{[f]_{x=x_{0}}}{2 \pi i} .
$$

Theorem 7.13. Let $f \in \mathcal{S}^{\prime}$ have the distributional jump behavior at $x=x_{0}$ of order n. Suppose that

$$
f(x)=O\left(|x|^{\alpha}\right) \quad(\mathrm{C}, m), \quad \text { as }|x| \rightarrow \infty .
$$


Then for any decomposition $\hat{f}=\hat{f}_{-}+\hat{f}_{+}$, where supp $\hat{f}_{-} \subseteq(-\infty, 0]$ and $\operatorname{supp} \hat{f}_{+} \subseteq[0, \infty)$. We have that the following convolutions are locally bounded functions and

$$
\left(e^{ \pm i x_{0} t} \hat{f}_{ \pm}( \pm t) * t_{+}^{k+1}\right)(x) \sim \pm[f]_{x=x_{0}} \frac{|x|^{k+1}}{i} \log x, \quad \text { as } x \rightarrow \infty,
$$

in the ordinary sense, where $k=\max \left\{m, n,\left[n+\alpha+\frac{1}{2}\right],\left[m+\alpha+\frac{1}{2}\right]\right\}$.

For the case of symmetric jumps of Fourier series we have the following result.

Theorem 7.14. Let $f \in \mathcal{S}^{\prime}$ be a $2 \pi$-periodic distribution having the following Fourier series

$$
\frac{a_{0}}{2}+\sum_{n=1}^{\infty}\left(a_{n} \cos n x+b_{n} \sin n x\right)
$$

If $f$ has a symmetric jump behavior at $x=x_{0}$ of order $k \geq 1$, then for any $\beta>k$

$$
\lim _{x \rightarrow \infty} \frac{1}{\log x} \sum_{n=1}^{\infty}\left(a_{n} \sin n x_{0}-b_{n} \cos n x_{0}\right)\left(1-\frac{n}{x}\right)^{\beta}=-\frac{1}{\pi}[f]_{x=x_{0}} .
$$

Acknowledgement. The first author gratefully acknowledges support by the Louisiana State Board of Regents grant LEQSF(2005-2007)-ENH-TR21.

\section{REFERENCES}

[1] Bremermann, H., Distributions, Complex Variables and Fourier Transforms, AddisonWesley, Reading, Massachusetts, 1965.

[2] Denjoy, A., Sur l'intégration des coefficients différentiels d'ordre supérieur, Fund. Math., 25 (1935), 237-320.

[3] Bochner, S. and Chadrasekharan, K., Fourier transforms, Annals of Mathematics Studies, No. 19, Princeton University Press, Princeton, N.J., 1949.

[4] Bochner, S., Lectures on Fourier Integrals, Annals of Mathematics Studies, No. 42, Princeton University Press, Princeton, N.J., 1959.

[5] Campos Ferreira, J., Introduction to the theory of distributions, Longman, Harlow, 1997.

[6] Estrada, R., Characterization of the Fourier series of a distribution having a value at a point, Proc. Amer. Math. Soc., 124 (1996), 1205-1212.

[7] Estrada, R., The Cesàro behaviour of distributions, Proc. Roy. Soc. London Ser. A, 454 (1998), 2425-2443.

[8] Estrada, R., A Distributional Version of the Ferenc-Lukács Theorem, Sarajevo J. Math., 1 (2005), 1-17.

[9] Estrada, R. and Kanwal, R. P., A Distributional Approach to Asymptotics. Theory and Applications, second edition, Birkhäuser, Boston, 2002.

[10] Estrada, R. and Vindas, J., Determination of jumps of distributions by differentiated means, Acta Math. Hungar., 124 (2009), 215-241. 
[11] Fejér, L., Über die Bestimmung des Sprunges der Funktion aus ihrer Fourierreihe, J. Reine Angew. Math., 142 (1913), 165-188.

[12] González Vieli, F. J., Pointwise Fourier inversion of distributions, Anal. Theory Appl., 24 (2008), 87-92.

[13] Gordon, R. A., The integrals of Lebesgue, Denjoy, Perron and Henstock, A.M.S., Providence, 1994.

[14] Gronwall, T. H., Über eine Summationsmethode und ihre Anwendung auf die Fouriersche Reihe, J. Reine Angew. Math., 147 (1916), 16-35.

[15] Hardy, G. H., Divergent Series, Clarendon Press, Oxford, 1949.

[16] Hardy, G. H. and Littlewood, J. E., Solution of the Cesàro summability problem for power series and Fourier series, Math. Z., 19 (1923), 67-96.

[17] Hardy, G. H. and Littlewood, J. E., The Fourier series of a positive function, J. London Math. Soc., 1 (1926), 134-138.

[18] Hardy, G. H., and Riesz, M., The general theory of Dirichlet's series, Cambridge Tracts in Mathematics and Mathematical Physics, No. 18, Cambridge University Press, Cambridge, 1952.

[19] Hardy, G. H. and Rogosinski, W. W., Fourier series, second edition, Cambridge Tracts in Mathematics and Mathematical Physics, no. 38, Cambridge, At the University Press, 1950.

[20] Hobson, E. W., The theory of functions of a real variable and the theory of Fourier's series, Vol. II, Dover Publications, New York, 1958.

[21] Łojasiewicz, S., Sur la valuer et la limite d'une distribution en un point, Studia Math., 16 (1957), 1-36.

[22] Lukács, F., Über die Bestimmung des Sprunges einer Funktion aus ihrer Fourierreihe, J. Reine Angew. Math., 150 (1920), 107-112.

[23] Marcinkiewicz, J., Sur les séries de Fourier, Fundamenta Mathematicae, 27 (1936), $38-69$.

[24] Móricz, F., Determination of jumps in terms of Abel-Poisson means, Acta. Math. Hungar., 98 (2003), 259-262.

[25] Móricz, F., Ferenc Lukács type theorems in terms of the Abel-Poisson means of conjugate series, Proc. Amer. Math. Soc., 131 (2003), 1243-1250.

[26] Pilipović, S., Stanković, B. and Takači, A., Asymptotic behaviour and Stieltjes Transformation of Distributions, Teubner-Texte zur Mathematik, Leipzig, 1990.

[27] Plancherel, M., Sur la convergence et sur la sommation par les moyennes de Cesàro

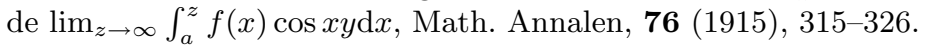

[28] Riesz, M., Une méthode de sommation équivalente à la méthode des moyennes arithmétiques, C. R. Acad. Sci. Paris, (1911), 1651-1654.

[29] Schwartz, L., Théorie des Distributions, Hermann, Paris, 1966.

[30] Seneta, E., Regularly Varying Functions, Lecture Notes in Mathematics, 598, Springer Verlag, Berlin, 1976.

[31] Titchmarsh, E. C., Introduction to the theory of Fourier Integrals, second edition, Clarendon Press, Oxford, 1948.

[32] de la Vallée Poussin, Ch. J., Sur l'approximation des fonctions d'une variable réelle et leurs dérivées par les polynômes et les suites limitées de Fourier, Bull. de l'Acd. Royale de Belgique, (1908), 193-254.

[33] Vindas, J., Structural Theorems for Quasiasymptotics of Distributions at Infinity, Pub. Inst. Math. (Beograd) (N.S.), 84(98) (2008), 159-174.

[34] Vindas, J., The structure of quasiasymptotics of Schwartz distributions, Linear and non-linear theory of generalized functions and its applications, Banach Center Publ. 88, Polish Acad. Sc. Inst. Math., Warsaw, 2010.

[35] Vindas, J. and Estrada, R., Distributionally Regulated Functions, Studia. Math., 181 (2007), 211-236. 
[36] Vindas, J. and Estrada, R., Distributional Point Values and Convergence of Fourier Series and Integrals, J. Fourier Anal. Appl., 13 (2007), 551-576.

[37] Vindas, J. and Estrada, R., On the jump behavior and logarithmic averages, J. Math. Anal. Appl., 347 (2008), 597-606.

[38] Vindas, J. and Estrada, R., On the support of tempered distributions, Proc. Edinb. Math. Soc. (2), 53 (2010), 255-270.

[39] Vindas, J. and Pilipović, S., Structural theorems for quasiasymptotics of distributions at the origin, Math. Nachr., 282 (2009), 1584-1599.

[40] Vladimirov, V. S., Drozhzhinov, Yu. N. and Zavialov, B. I., Tauberian Theorems for Generalized Functions, Kluwer Academic Publishers, Dordrecht, 1988.

[41] Vladimirov, V. S., Methods of the Theory of Generalized Functions, Taylor \& Francis, London, 2002.

[42] Walter, G., Pointwise convergence of distribution expansions, Studia Math., 26 (1966), 143-154.

[43] Walter, G., Fourier series and analytic representation of distributions, SIAM Review, 12 (1970), 272-276.

[44] Walter, G. and Shen, X., Wavelets and other orthogonal systems, second edition, Studies in Advanced Mathematics, Chapman \& Hall/CRC, Boca Raton, 2001.

[45] Zygmund, A., Sur un théorème de M. Gronwall, Bull. Acad. Polon., (1925), 207-217.

[46] Zygmund, A., Trigonometric Series, Vols. I \& II, second edition, Cambridge University Press, New York, 1959.

Department of Mathematics, Louisiana State University, Baton Rouge, LA 70803, U.S.A

E-mail address: jvindas@cage.Ugent.be, restrada@math.1su.edu 